\title{
Make Interactive Complex Heatmaps in $\mathbf{R}$
}

\author{
Zuguang Gu \\ German Cancer Research Center
}

\author{
Daniel Hübschmann \\ German Cancer Research Center
}

\begin{abstract}
Heatmap is a powerful visualization method on two-dimensional data to reveal patterns shared by subsets of rows and columns. In $\mathrm{R}$, there are many packages that make heatmaps. Among them, ComplexHeatmap provides rich tools for constructing highly customizable heatmaps. It can easily establish connections between information from multiple sources by automatically concatenating and adjusting multiple heatmaps as well as complex annotations, which makes it widely applied in data analysis in various fields, especially in Bioinformatics. Nevertheless, the limit of ComplexHeatmap still exists. It only generates static plots which restricts deeper inspections on complex heatmaps, e.g., to look into a subset of rows and columns when a specific pattern of interest is observed from the heatmap. In this work, we described a new R/Bioconductor package InteractiveComplexHeatmap that brings interactivity to ComplexHeatmap. InteractiveComplexHeatmap is designed with an easy-to-use interface where static complex heatmaps can be directly exported to an interactive Shiny web application only with one extra line of code. The interactive application contains comprehensive tools for manipulating heatmaps. Besides common tools as supported in other interactive heatmap packages, InteractiveComplexHeatmap additionally supports, e.g., selecting over multiple heatmaps and searching heatmaps via row or column labels. Also, InteractiveComplexHeatmap provides methods for exporting static heatmaps from other popular heatmap functions, e.g., heatmap.2() or pheatmap(), to interactive heatmap applications. Finally, InteractiveComplexHeatmap provides flexible functionalities for integrating interactive heatmap widgets into other Shiny applications. InteractiveComplexHeatmap provides a user interface for self-defining response to the selection events on heatmaps, which helps to implement more complex Shiny web applications.
\end{abstract}

Keywords: visualization, interactive heatmap, complex heatmap, Shiny, R.

\section{Introduction}

Heatmap is a popular visualization method on two-dimensional matrices where colors are the major aesthetic elements mapping to data (Wilkinson and Friendly 2009). In routine data analysis procedures, matrix for heatmap visualization is normally accompanied with row and column reordering, e.g., by hierarchical clustering or seriation (Hahsler, Hornik, and 
bioRxiv preprint doi: https://doi.org/10.1101/2021.03.08.434289; this version posted May 18, 2021. The copyright holder for this preprint (which was not certified by peer review) is the author/funder, who has granted bioRxiv a license to display the preprint in perpetuity. It is made available under aCC-BY-ND 4.0 International license.

Buchta 2008), so that features with similar patterns are grouped closely and they can be easily identified from the colors on heatmap. The reordering methods can also be chosen specifically for the topic under study. For example, the R package EnrichedHeatmap ( $\mathrm{Gu}$, Eils, Schlesner, and Ishaque 2018) can be used to visualize how DNA demethylation is enriched around the transcriptional start sites (TSS) of genes by a heatmap where columns are ordered by genomic distances from $\mathrm{CpG}$ dinucleotides to gene TSS and rows are ordered by the enrichment score defined by EnrichedHeatmap. Heatmap is widely applied in various research fields, especially popular in Bioinformatics. Since the first paper on heatmap visualization on a gene expression dataset was published (Eisen, Spellman, Brown, and Botstein 1998), heatmap has been a standard tool for visualizing e.g., gene expression, DNA methylation and other high-throughput datasets represented as matrices. In R (R Core Team 2020), there are several packages that implement heatmaps. The function heatmap() from package stats is the most fundamental one, but with very limited functionality. The function heatmap.2() from package gplots (Warnes, Bolker, Bonebakker, Gentleman, Huber, Liaw, Lumley, Maechler, Magnusson, Moeller, Schwartz, and Venables 2020) is an enhanced version of heatmap() which supports more graphics on heatmap, such as a color legend with value distribution and trace lines showing the difference of values to the column or row medians. There are also packages implemented with the grid graphics system such as the function pheatmap() from package pheatmap (Kolde 2019) and the function aheatmap() from package NMF (Gaujoux and Seoighe 2010). They provide more flexible controls on heatmaps.

As data emerges fast in dimensions nowadays, especially in the Genomics field, an efficient visualization for integrative analysis or multi-omics analysis is urgently needed to associate multiple types of data for easily revealing relationships between multiple objects. From the aspects of heatmap visualization, it reflects in two points. The first is the support of heatmap annotations which contain extra information to associate to the main heatmap. For example, in a typical heatmap visualization on gene expression data where rows are genes and columns are patients, it is common that patients have clinical meta-data available, such as age, gender or whether the patient has certain DNA mutations. With annotation attached to heatmap, it is easy to identify, e.g., whether a group of genes showing high expression correlate to a certain age interval or whether they have specific types of DNA mutations. heatmap() and heatmap.2() only support single heatmap-like annotation for one numeric or character vector. pheatmap () and aheamtap () allow multiple heatmap-like annotations for corresponding more information to heatmap. The package superheat (Barter and Yu 2018) and heatmap3 (Zhao, Yin, Guo, Sheng, and Shyr 2021) support more types of graphics for annotations, such as points or lines which are able to make more accurate visual representation on annotation data. The second point of visualizing multiple sources of information is to directly apply "complex heatmap visualization" by simultaneously linking multiple heatmaps to make it straightforward to compare patterns between heatmaps. For example, in our published study (Gu et al. 2018), we applied complex heatmap visualization on gene expression, DNA methylation and various histone modifications to reveal general transcriptional regulation patterns among multiple human tissues. To implement both complex annotation and heatmap visualization, we previously developed an advanced heatmap package ComplexHeatmap (Gu, Eils, and Schlesner 2016a). It supports not only the basic annotation graphics as in other packages, but also a variety of extra complex annotation graphics such as violin plot or horizon plot, and it even allows users to self-define their own annotation graphics. ComplexHeatmap provides a simple syntax to link multiple heatmaps and annotations where rows or columns 
of all heatmaps are adjusted simultaneously. The simplicity of its user interface and comprehensiveness of its functionalities make ComplexHeatmap widely used in Bioinformatics to reveal interesting patterns from data that are potentially biologically meaningful.

After a specific pattern of subset of rows and columns is observed from the heatmap, the next step is to extract corresponding rows and columns for downstream analysis, which requires interactivity on heatmaps. There are also several $\mathrm{R}$ packages that implement interactive heatmaps such as d3heatmap (Cheng and Galili 2018), heatmaply (Galili, O'Callaghan, Sidi, and Sievert 2017) and iheatmapr (Schep and Kummerfeld 2017). The interactivity allows hovering on heatmap cells and selecting a region from it. There are other web-based and non-R implementations, such as Morpheus (Morpheus 2021), Heatmapper (Babicki, Arndt, Marcu, Liang, Grant, Maciejewski, and Wishart 2016), Clustergrammer (Fernandez, Gundersen, Rahman, Grimes, Rikova, Hornbeck, and Ma'ayan 2017) and NG-CHM (Ryan, Stucky, Wakefield, Melott, Akbani, Weinstein, and Broom 2019) which allow users to directly upload data to the web server and manipulate heatmap with no programming knowledge.

ComplexHeatmap is popular and powerful for generating static complex heatmaps. Here we developed a new R/Bioconductor package InteractiveComplexHeatmap that brings interactivity to ComplexHeatmap. InteractiveComplexHeatmap can be very easily applied that any static complex heatmap generated by ComplexHeatmap can be directly exported to an interactive Shiny web application only with one extra line of code. The interactive application contains comprehensive tools for manipulating heatmaps. Besides common tools as supported in other interactive heatmap packages, InteractiveComplexHeatmap additionally supports, e.g., selecting over multiple heatmaps or searching heatmaps via row or column labels. The latter would be especially useful when users already have some features of interest to look into. Also, InteractiveComplexHeatmap provides methods to export static heatmaps from other popular heatmap functions, e.g., heatmap.2() or pheatmap(), to interactive heatmap applications, which greatly expands the ability of interactive heatmap visualization in R. Finally, InteractiveComplexHeatmap provides functionalities for integrating interactive heatmap widgets into other Shiny applications. InteractiveComplexHeatmap provides a user interface for selfdefining response to the selection events on heatmaps, e.g., by clicking or brushing, which helps to develop more complex Shiny web applications. InteractiveComplexHeatmap can be freely obtained from https://bioconductor.org/packages/InteractiveComplexHeatmap/.

The paper is structured as follows. In Section 2, we first made a brief introduction to the functionalities in ComplexHeatmap. In Section 3, we described how to export static heatmaps to an interactive Shiny web application and we also described the tools provided by the interactive application. In Section 4, we explained how the interactivity is implemented in InteractiveComplexHeatmap. In Section 5, we explained in detail how to integrate InteractiveComplexHeatmap in Shiny application development. And finally, in Section 6, as case studies, we implemented a complex interactive heatmap application which visualizes results of differential gene expression analysis and we proposed an approach for interactively visualizing two-dimensional density distributions with heatmaps.

\section{A brief introduction to ComplexHeatmap}

In this section, we briefly introduce basic functionalities of making single heatmaps and a list of heatmaps with ComplexHeatmap. For more comprehensive usages, readers are recommended 
to refer to the ComplexHeatmap complete reference ( $\mathrm{Gu} 2021$ ).

ComplexHeatmap is implemented with the grid graphics engine under S4 object-oriented system. There are three major classes defined in the package: a Heatmap class that defines a single heatmap, a HeatmapList class that defines a list of heatmaps, and a HeatmapAnnotation class that defines a list of heatmap annotations.

\subsection{A single heatmap}

The function Heatmap ( ) makes a single heatmap and returns an object in Heatmap class. The only mandatory argument in Heatmap () is a matrix, either numeric or character. It provides numerous additional arguments for customizing heatmap. Besides common functionalities also available in other heatmap functions, Heatmap() has following major features:

- Flexible controls of clustering. The hierarchical clustering accompanied with heatmap can be specified in various ways, i.e., 1 . by a predefined distance method such as "euclidean" or "pearson", 2. by a distance function that calculates pairwise distance between two vectors or directly from a matrix, 3 . by a clustering object e.g. a hclust or a dendrogram object or an object that can be coerced to dendrogram by a proper as . dendrogram () function, or 4 . by a clustering function that takes matrix as input and returns a dendrogram object. Besides that, dendrograms can be rendered on both edges and nodes, e.g., to assign different colors for dendrogram branches or to add symbols on dendrogram nodes (Figure 1A).

- Split heatmap. Heatmap splitting is an efficient way to highlight group-wise patterns (Figure 1D). ComplexHeatmap provides various ways for splitting heatmap into "slices" on both rows and columns: 1 . Set a number of groups for $k$-means clustering; 2 . Set a categorical variable which can be a vector or a data frame, then the heatmap is split by all combinations of levels in the categorical variable; 3 . If hierarchical clustering is already applied, the splitting can be specified as a single number so that cutree () is internally applied to split. For the first two splitting methods, if clustering is turned on, hierarchical clustering is performed within each heatmap slice, also a second clustering is performed over heatmap slices based on the slice means.

- Flexible controls of colors and legends. It allows exact mapping between colors and values in the matrix with a color mapping function by specifying breaks and corresponding colors, then remaining colors are linearly interpolated in the corresponding intervals. It also allows flexible configurations on heatmap legends, such as multiple-color scheme legends. Please refer to Section "Legends" in ComplexHeatmap complete reference (Gu 2021) for more examples.

- Render heatmap body as a raster image. For a heatmap on a huge matrix that is saved as vector graphics (e.g., a pdf figure), rasterization helps to efficiently reduce the final file size while the loss of figure quality is ignorable. Heatmap() supports various methods for image rasterization. See detailed explanations and comparisons in ( $\mathrm{Gu} 2020$ ).

- Customize heatmap. There are two ways for customizing heatmap with user-defined code: 1 . to customize heatmap body via cell_fun or layer_fun argument to add self-defined graphics to heatmap cells when heatmap is drawing (Figure 1B), and 2. to use decorate_*() family functions, e.g., decorate_annotation(), to add graphics to any heatmap component after the heatmap is drawn. 
bioRxiv preprint doi: https://doi.org/10.1101/2021.03.08 434289; this version posted May 18, 2021. The copyright holder for this preprint (which was not certified by peer review) is the author/funder, who has granted bioRxiv a license to display the preprint in perpetuity. It is made available under aCC-BY-ND 4.0 International license.

A

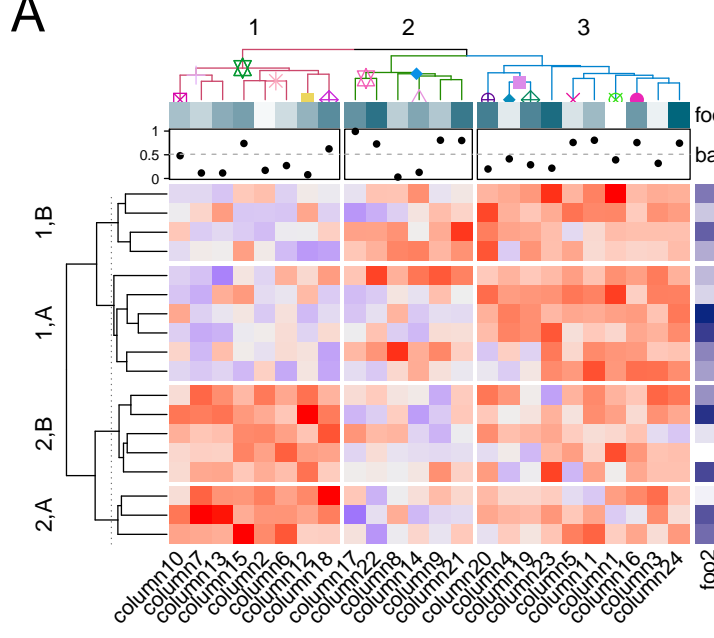

C

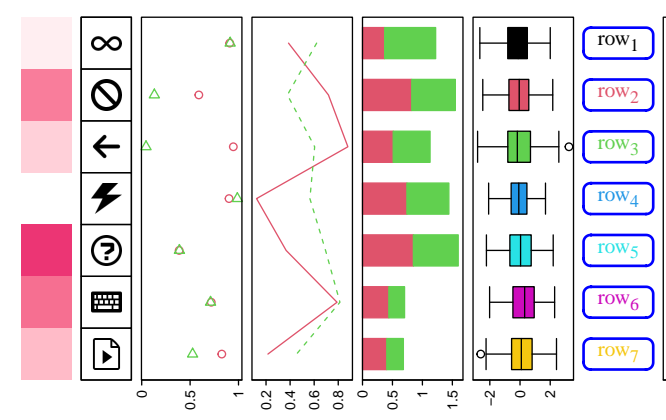

B

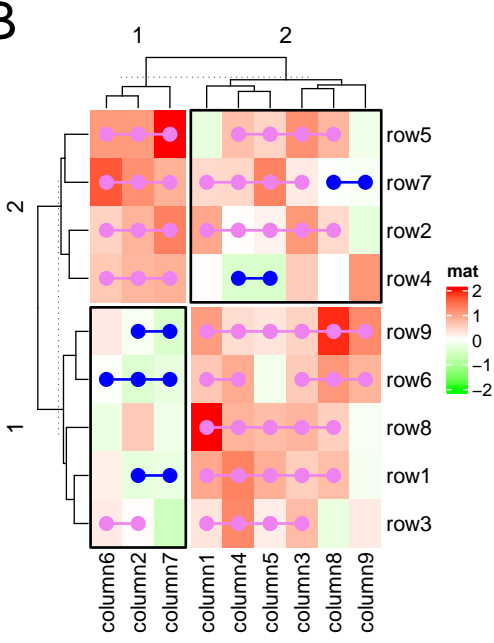

D

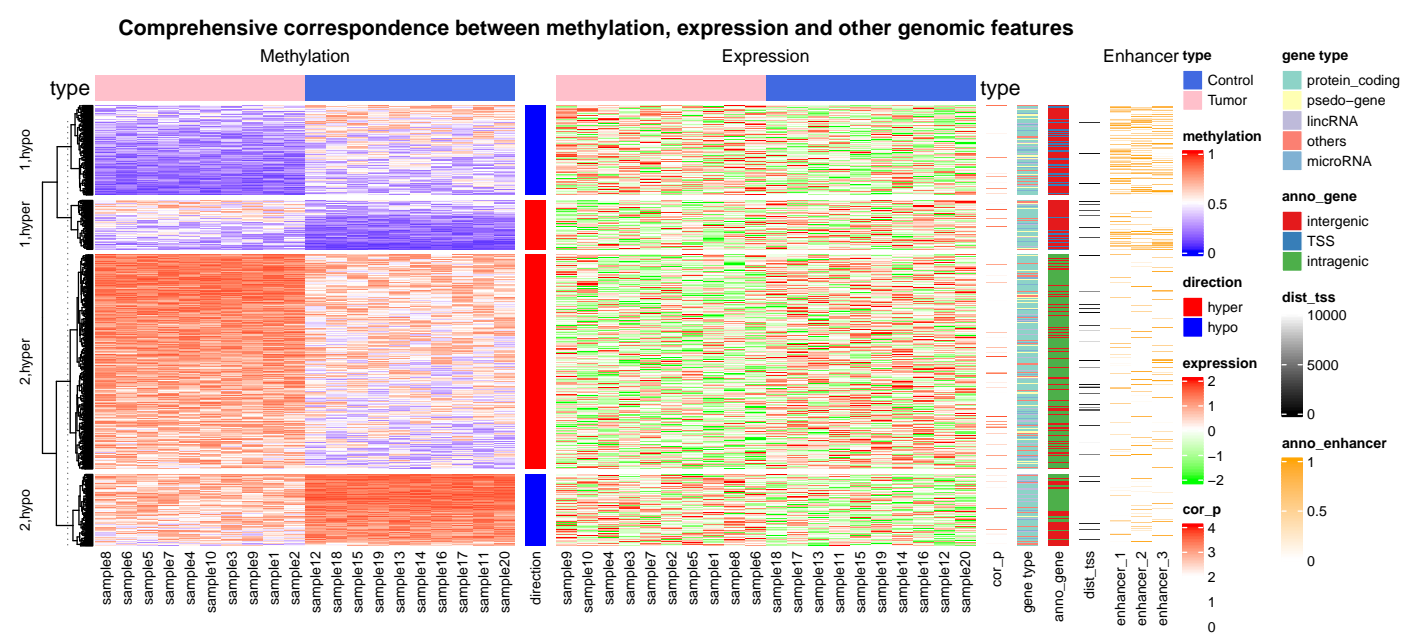

Figure 1: Demonstration of ComplexHeatmap. A) A heatmap with both row and column annotations. The columns on the heatmap were split by a three-group $k$-means clustering and rows were split by combination of a categorical variable and a two-group $k$-means clustering. B) A heatmap with customizations. On the heatmap, horizontal neighbour cells were connected if they had the same sign. Black borders were added to the top right and bottom left heatmap slices. C) Examples of various annotation graphics supported in ComplexHeatmap. D) An example of complex heatmap visualization based on a real-world dataset. 


\subsection{Heatmap annotations}

Heatmap annotations are important components of a heatmap that show additional information associated with rows and columns. ComplexHeatmap provides flexible support for annotations and defining new annotation graphics. Annotations can be put on the four sides of the heatmap and they are automatically reordered and split according to the heatmap.

There are following annotation graphics demonstrated in Figure 1C (from left to right):

- Heatmap-like annotation. It is named as "simple annotation" in ComplexHeatmap. It visualizes a vector or a matrix, either numeric or character.

- Image annotation. It supports images in various formats, e.g., png, svg, pdf, jpg.

- Points annotation. It supports a single numeric vector or a numeric matrix.

- Lines annotation. It supports a single numeric vector or a numeric matrix. Additionally, it supports to perform loess smoothing over data points.

- Barplot annotation. It also supports stacked bar plots.

- Boxplot annotation.

- Text annotation. It supports constructing more customized text with package gridtext (Wilke 2020).

- Histogram annotation.

- Violin annotation. It visualizes a list of distributions. Alternatively, the distributions can also be visualized by normal density plots or heatmaps.

- Joy plot annotation.

- Horizon plot annotation.

All built-in annotation graphics are implemented by annotation functions named with anno_ prefix, e.g., anno_points () for points annotation. Besides the above listed annotations, ComplexHeatmap supports more complex annotations. For example, there is a "mark annotation" by anno_mark() which draws labels for a subset of rows or columns where the labels are shifted from their original positions to get rid of overlapping and lines are drawn to connect labels to their corresponding rows or columns. ComplexHeatmap provides a user interface for creating new annotation graphics. For example, package EnrichedHeatmap (Gu et al. 2018) defined an "enriched annotation" by anno_enriched() which visualizes average enrichment of certain genomic signals over a list of genomic features, and package simplifyEnrichment (Gu and Hübschmann 2021) defined a "word cloud annotation" by anno_word_cloud() which visualizes summaries of groups of rows by word clouds.

The function HeatmapAnnotation() accepts multiple annotations specified as name-value pairs. Simple annotations are specified as vectors, matrices or a data frame and legends are automatically generated for them. Other annotations should be specified via functions anno_*(). An example is as follows.

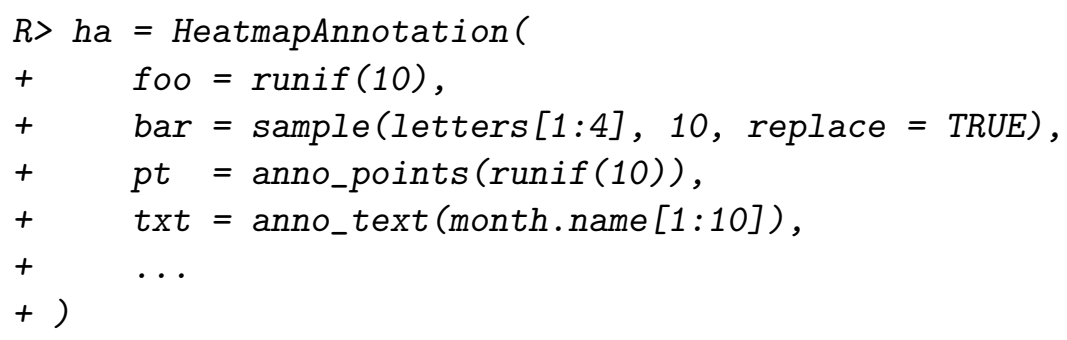


Row annotations should be set with one additional argument which = "row" or by the helper function rowAnnotation(). Column annotations are assigned to argument top_annotation or bottom_annotation and row annotations are assigned to argument left_annotation or right_annotation in Heatmap().

\subsection{A list of heatmaps}

The main feature of ComplexHeatmap is that it supports concatenating multiple heatmaps and annotations so that it is possible to visualize associations between various sources of information. ComplexHeatmap provides a simple syntax for concatenating heatmaps with the operator + . The expression returns a HeatmapList object and directly printing the HeatmapList object draws the heatmap.

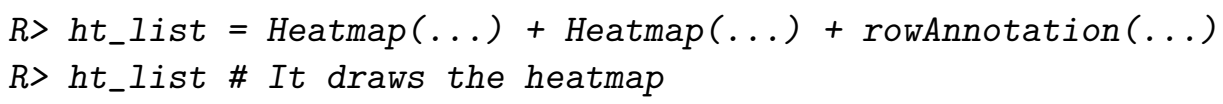

We previously introduced annotations as components of a single heatmap. Here row annotations can also be independently concatenated to the heatmap list, as are demonstrated in the above code. Alternatively, which is less used, the heatmap lists can be vertically concatenated with the operator $\%$ v .

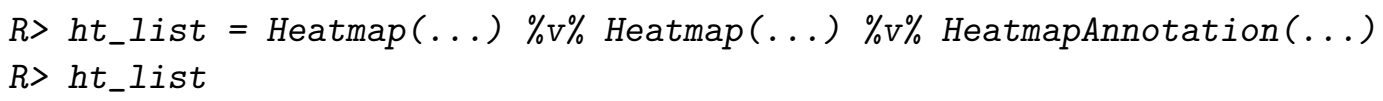

The amount of heatmaps and annotations to be concatenated can be arbitrary. The ordering and splitting of all heatmaps are adjusted by the main heatmap, which is by default the first numeric matrix, or other heatmap in the list that is specified by the user.

The function draw () should be explicitly applied to the heatmap object when the heatmap is drawn in a non-interactive environment, such as inside a function, inside a for/if-else block, or in an R script running under command line. Moreover, draw() provides arguments for fine-tuning heatmap, such as adding self-defined legends and adjusting spaces between heatmaps.

$R>\operatorname{draw}\left(h t_{-}\right.$list, ...)

\subsection{High-level plots implemented with ComplexHeatmap}

The flexibility of ComplexHeatmap allows users to implement new high-level graphics on data with a matrix-like structure. In ComplexHeatmap, there are following high-level graphics functions that have already been implemented:

- densityHeatmap(). It visualizes a list of distributions via heatmap where the density values are mapped to colors.

- oncoPrint(). It visualizes multiple genomic alteration events (e.g. single-base mutations or fragment deletions) in a list of genes and in multiple patients. 
- UpSet(). It visualizes intersections over multiple sets. This is an enhanced version of package UpSetR (Conway, Lex, and Gehlenborg 2017). UpSet () is also able to visualize intersections over genomic intervals.

More importantly, the three functions all return Heatmap objects, thus, the graphics can be concatenated to additional heatmaps or annotations to construct a complex view of the data. As an example, an oncoPrint can be concatenated to a gene expression heatmap to quickly connect the relationship between DNA mutations and the influence on gene expression. Example code is simply as follows:

$R>\operatorname{oncoPrint}(\ldots)+\operatorname{Heatmap}(\ldots)$

\subsection{A complex example}

Figure 1D demonstrates complex heatmap visualization on a dataset randomly generated but based on patterns found in an unpublished work. The figure visualizes associations between DNA methylation, gene expression, enhancers and gene-related information. In heatmaps, each row corresponds to a differentially methylated region (DMR, which is a genomic region showing significantly different methylation between tumor and control samples) or other objects that are associated with the corresponding DMR. In Figure 1D, there are following heatmaps from left to right:

1. A heatmap of methylation levels in DMRs. Red corresponds to $100 \%$ methylation in the DMR and blue corresponds to no methylation.

2. A one-column heatmap showing the direction of differential methylation. hyper means methylation is higher in tumor samples than in control and hypo means methylation is lower in tumor samples.

3. A heatmap of gene expression. Each gene is the nearest gene in the genome of the associated DMR. Note only the DMRs with methylation levels negatively correlated to the expression level of their nearest genes are kept in Figure 1D.

4. A one-column heatmap of p-values from Pearson correlation test between gene expression and methylation.

5. A one-column heatmap of the gene type, i.e., whether the corresponding gene is a protein-coding gene or of other type?

6. A one-column heatmap of the annotation of DMRs to genes, i.e., does the DMR locate in gene TSS or outside gene regions?

7. A one-column heatmap of the genomic distance between DMR to TSS of the associated gene.

8. A heatmap showing the overlap between enhancers and DMRs. Enhancers are genomic regions where proteins can bind to regulate gene expression.

The heatmap list is split by the combination of directions of differential methylation and a two-group $k$-means clustering. The latter is to distinguish high-methylation group and low methylation group. In Figure 1D, the complex heatmaps reveal that highly methylated DMRs are enriched in intergenic and intragenic regions and rarely overlap with enhancers (row group "2, hypo" and "2, hyper"), while in contrast, lowly methylated DMRs are enriched in TSS and 
enhancers (row group "1, hypo" and "1, hyper"). This might imply that enhancers associate with low methylation and methylation changes in enhancers might affect their transcriptional activities on related genes.

\section{Export heatmap to a Shiny web application}

\subsection{The function htShiny()}

For any single heatmap or a list of heatmaps represented as a Heatmap or a HeatmapList object produced by ComplexHeatmap, the function htShiny() in InteractiveComplexHeatmap can be simply applied to export it to a Shiny web application (Chang, Cheng, Allaire, Sievert, Schloerke, Xie, Allen, McPherson, Dipert, and Borges 2021). To demonstrate the usage, we generated a list of two heatmaps of a numeric heatmap and a character heatmap. In the numeric heatmap, $k$-means clustering with two groups was applied on both rows and columns. A point annotation was put on top of the first heatmap as a heatmap component and a barplot annotation was inserted between the first and the second heatmaps.

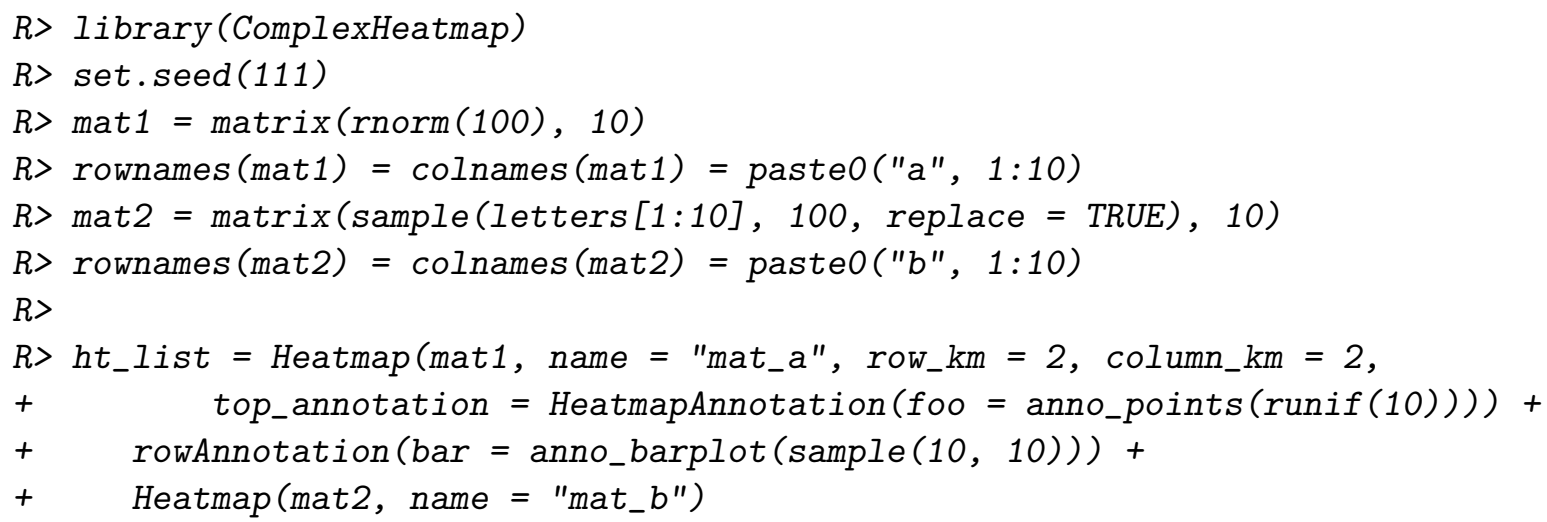

After the heatmap object ht_list is created, it can be directly sent to htShiny(). The interactive heatmap application is automatically opened in a web browser or in a pop-up window in RStudio IDE (Figure 2).

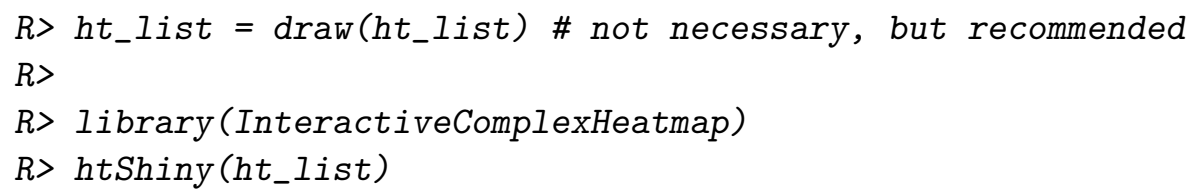

To use htShiny(), the heatmap object is recommended to be updated with the function draw(). If it has not been updated, it will be applied inside htShiny() automatically. Updating by draw() speeds up loading the Shiny application because draw() applies clusterings which is normally the most time-consuming step in heatmap generation. After draw () is executed, clustering results are saved in the returned heatmap object so that repeatedly drawing heatmap can directly use the saved clustering results. If the heatmap includes randomness, such as $k$-means clustering by setting row_km or column_km argument, it is necessary to execute $\operatorname{draw}()$ before sending the heatmap object to htShiny() to get rid of obtaining different heatmaps when executing htShiny() multiple times. 
Executing the function htShinyExample() with no argument prints a list of 54 examples of various usages of interactive heatmaps. Specifying an index in htShinyExample() runs the corresponding interactive application as well as showing the source code that generates the application, e.g., htShinyExample(1.5) demonstrates an example of exporting a list of two heatmaps to an interactive web application.

\subsection{Tools in the interactive heatmap application}

In the Shiny application illustrated in Figure 2, there are three main components: the original heatmap (labelled as "A" in Figure 2), the selected sub-heatmap (labelled as "B" in Figure 2) and the output (labelled as "C" in Figure 2) that prints information of the heatmap cell that was clicked or the sub-heatmap that was selected. In the original heatmap, users can click on it or select an area from it. If an area is selected from the original heatmap, a sub-heatmap is drawn on the right side of the application. Both heatmaps can be resized by dragging from their bottom right.

Under the original heatmap, there are various tools integrated:

- Search heatmap: If the corresponding matrices have row or column labels, it allows users to search matrix labels to obtain a subset of rows and columns. The keywords can be exactly matched to row or column labels or be a regular expression. Once the corresponding rows or columns are found in heatmaps, a sub-heatmap is drawn in the right sub-heatmap component.

- Configure brush: It configures visual style of the brush which selects an area from heatmap, i.e., border color and background color, border width and opacity of the brush.

- Save image: The main heatmap can be saved into a file in one of the three formats: png, pdf and svg.

- Resize image: The size of heatmap can be precisely controlled by manually providing a value for it.

Under the sub-heatmap, there are also tools for controlling the sub-heatmap.

- Configure sub-heatmap: There are three sections of controls. 1. Basic controls such as whether to show row or column labels, 2. Brushing on the original heatmap might not precisely capture rows or columns that users expected. Users can manually remove a certain number of rows or columns from four dimensions of the selected sub-heatmap. 3. Selected sub-heatmap can be further converted into a second interactive heatmap application. It will be introduced in more detail in Section 3.4.

- Export table: The values in sub-heatmap can be viewed and exported as a text table. The table also includes values in corresponding annotations.

- Save sub-heatmap: The sub-heatmap can be saved into a file in one of the three formats: png, pdf and svg. 
bioRxiv preprint doi: https://doi.org/10.1101/2021.03.08.434289; this version posted May 18, 2021. The copyright holder for this preprint (which was not certified by peer review) is the author/funder, who has granted bioRxiv a license to display the preprint in perpetuity. It is made available under aCC-BY-ND 4.0 International license.
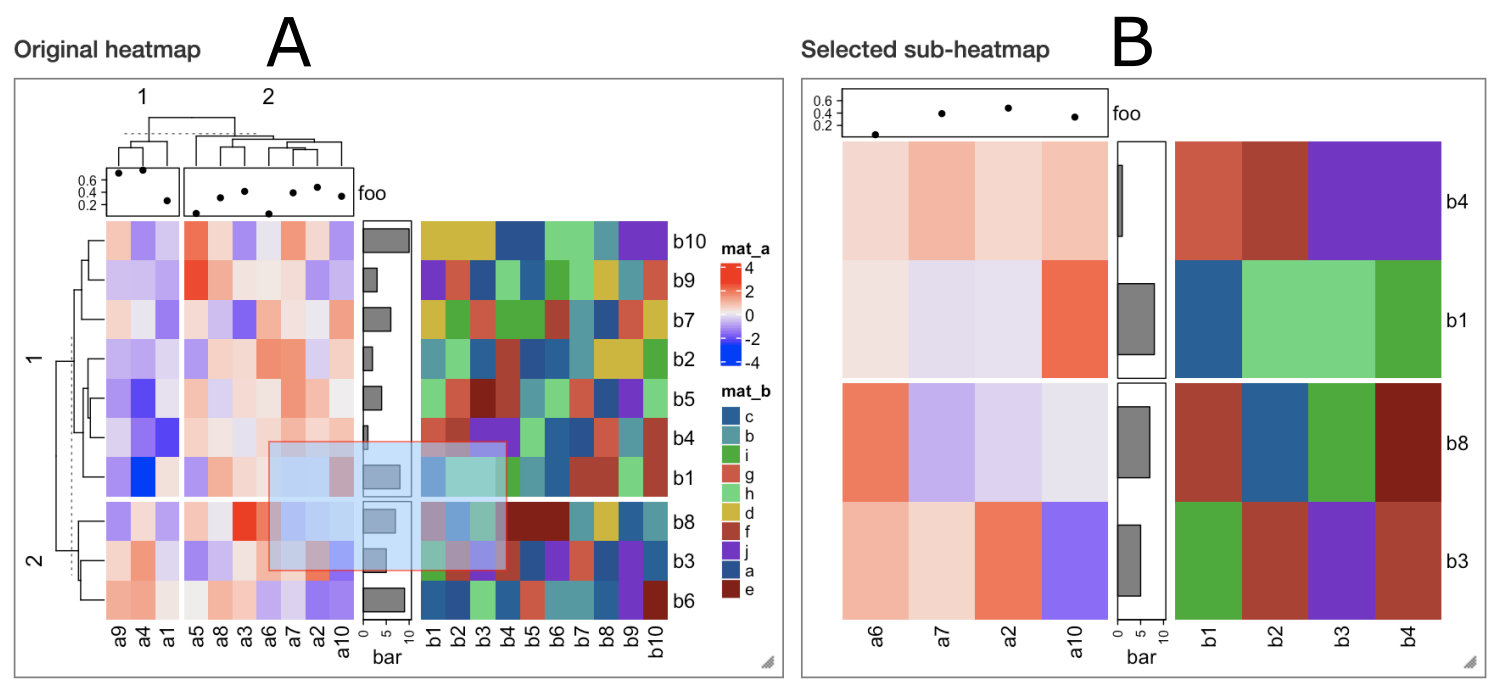

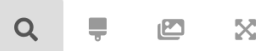

田曲恕

\section{Keywords}

Multiple keywords separated by ',' $\square$ Regular expression

Which dimension to search?

on rows $\bigcirc$ on columns

Which heatmaps to search?

$\checkmark$ mat_a

$\checkmark$ mat_b

Extend sub-heatmap to all heatmaps and annotations?

$\square$ yes

Search

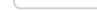

Output $C$

Selected over 2 heatmaps with 4 rows and 8 columns. Row and column indices can be obtained by copying following code:

show/hide code

Figure 2: A demonstration of interactive complex heatmap. On the left is the original heatmap (labelled as "A") and on the right is the sub-heatmap (labelled as "B") that was selected from left. Below both heatmaps are the tools for controlling heatmaps. At the bottom of the application is the output (labelled as "C") that shows the information of the heatmap cell that was clicked or the sub-heatmap that was selected. 
bioRxiv preprint doi: https://doi.org/10.1101/2021.03.08.434289; this version posted May 18, 2021. The copyright holder for this preprint (which was not certified by peer review) is the author/funder, who has granted bioRxiv a license to display the preprint in perpetuity. It is made available under aCC-BY-ND 4.0 International license.

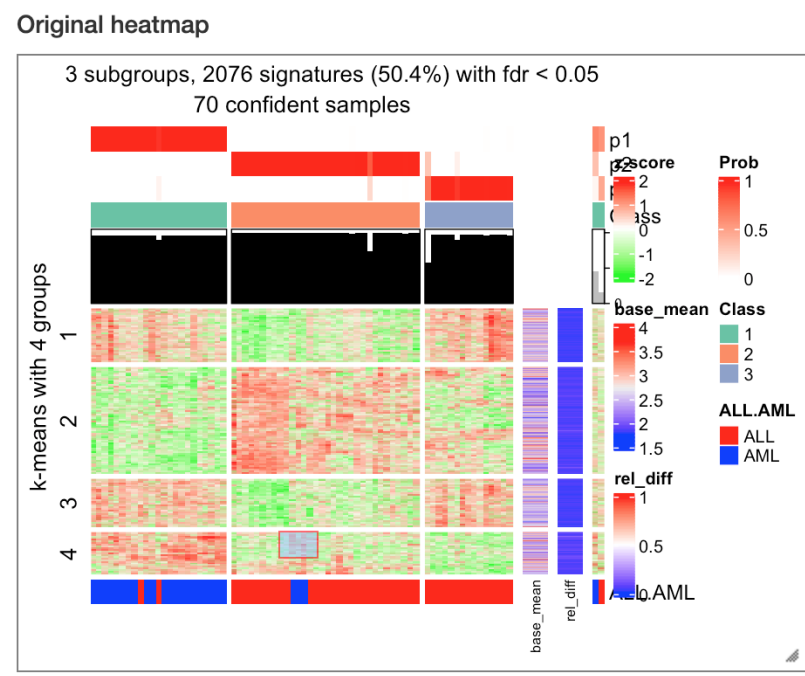

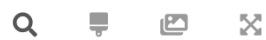

Selected sub-heatmap

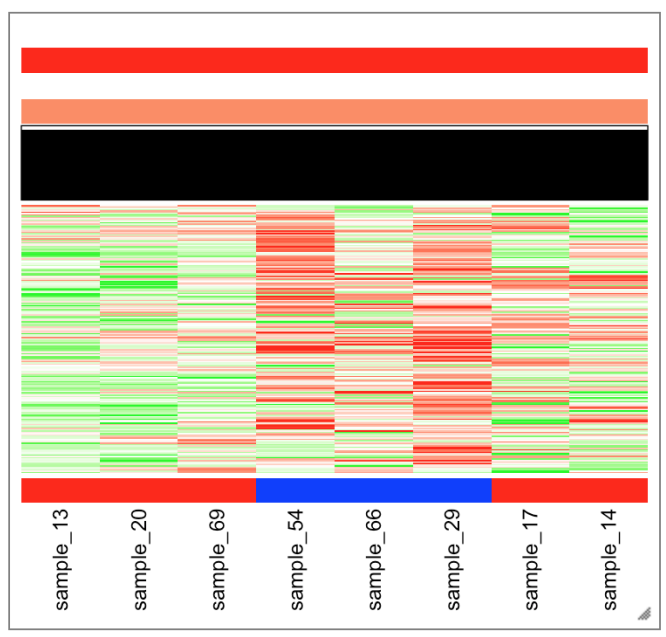

田迸品

Figure 3: Interactivating last generated heatmap. The original heatmap is generated by function get_signatures() from package cola.

- Resize sub-heatmap: The size of sub-heatmap can be precisely controlled by manually providing a value for it.

At the bottom of the application, there is an output component. If a heatmap cell was clicked, the output component prints the meta information of that cell, such as the value, the row and column indices and labels. If there are annotations associated in heatmap, corresponding annotation values are also printed. If an area was selected from the original heatmap, the output component prints a runnable code that can be used to obtain row and column indices from the corresponding matrices. The output component can be self-defined to allow more complex output to respond to user's actions on the heatmap. It will be introduced in more detail in Section 5.

\subsection{Use last generated heatmaps}

ComplexHeatmap is broadly used in many scripts and packages where they generate heatmaps but do not directly return Heatmap/HeatmapList objects. Nevertheless, this won't affect the use of InteractiveComplexHeatmap because the last generated heatmap object is always automatically saved. Calling htShiny() without a heatmap object will automatically use the last one. We demonstrate this functionality with the package cola $(\mathrm{Gu}$, Schlesner, and Hübschmann 2020).

cola heavily uses ComplexHeatmap to implement various customized heatmaps to visualize consensus clustering results as well as results from downstream analysis. As an example, the function get_signatures() extracts signatures that are significantly different between the predicted subgroups. get_signatures() makes a list of heatmaps for visualizing the patterns of signatures and returns a data frame of the signatures as well as various statistics for the statistical test, thus, we cannot directly interact with the heatmap object. When 
get_signatures () draws the heatmaps, the heatmap object is saved internally, then directly calling htShiny () without any argument converts the signature heatmap into an interactive application (Figure 3).

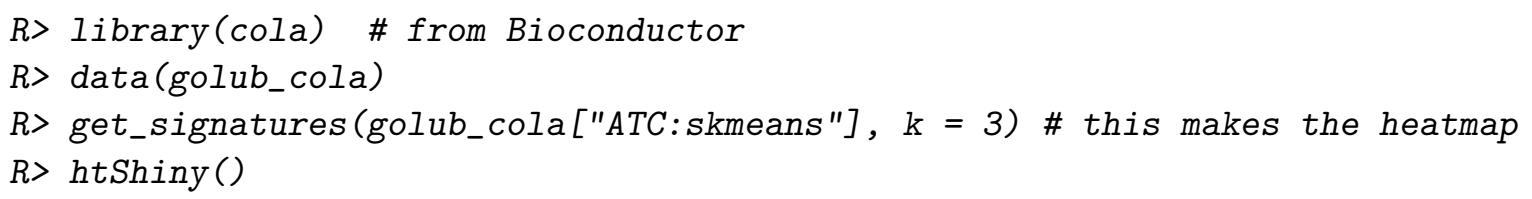

Note the functionality of automatically saving the last heatmap object is only turned on when InteractiveComplexHeatmap is loaded, which means, library (InteractiveComplexHeatmap) should be executed before making heatmap, or users can manually turn it on by executing ComplexHeatmap: : ht_opt (save_last $=$ TRUE).

\subsection{Recursive interactive heatmap application}

When the original heatmap visualizes a huge matrix, in the interactive heatmap application, a small selection rectangle would cover a dense subset of rows and columns where single cells are still hard to identify in the sub-heatmap component. In this case, the sub-heatmap can be continually exported to another independent interactive heatmap widget which is in a new layer above current one, just by clicking the button "Interactivate sub-heatmap" in the tool under sub-heatmap (Figure 4). This process can be recursively applied until users are satisfied with the details seen in the sub-heatmap.

\subsection{Applications on other heatmap functions}

InteractiveComplexHeatmap automatically interactivates static heatmaps generated by ComplexHeatmap. To facilitate analysis from users who still use other heatmap functions and also want to convert their heatmaps into interactive applications, in the companion package ComplexHeatmap, we implemented three "translation functions": ComplexHeatmap: : : heatmap (), ComplexHeatmap: : :heatmap.2() and ComplexHeatmap: :pheatmap() to replace stats: :heatmap(), gplots : : heatmap. 2() and pheatmap: : pheatmap (). The three translation functions have the same set of arguments as original ones and corresponding arguments are translated to proper settings in ComplexHeatmap so that they make heatmaps visually as same as possible to original heatmaps. Note in order not to dilute user's namespace, heatmap() and heatmap.2() are not exported from ComplexHeatmap and users must add ComplexHeatmap : : : prefix to use them. While for pheatmap(), if package pheatmap is already loaded ahead of ComplexHeatmap, there is a message printed to inform ComplexHeatmap::pheatmap() overwrites pheatmap: : pheatmap(). Since the translation functions have the same sets of arguments as original ones, no further modification on code that users need to apply.

The three functions ComplexHeatmap: : :heatmap(), ComplexHeatmap:: :heatmap.2() and ComplexHeatmap: : pheatmap () all generate Heatmap objects, thus heatmaps from these three functions can be converted into interactive applications. The returned object by e.g., ComplexHeatmap: : : heatmap() can be sent to htShiny(), or htShiny() can be directly called with no argument after the heatmap is drawn. Example code is as follows:

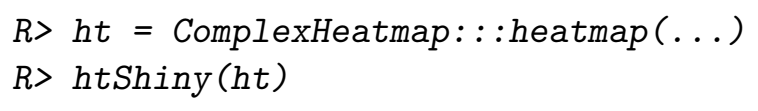


bioRxiv preprint doi: https://doi. org/10.1101/2021.03.08 434289- this version posted May 18,2021 . The copyright holder for this preprint (which was not certified by peer review) is the author/funder, who has granted bioRxiv a license to display the preprint in perpetuity. It is made available under aCC-BY-ND 4.0 International license.
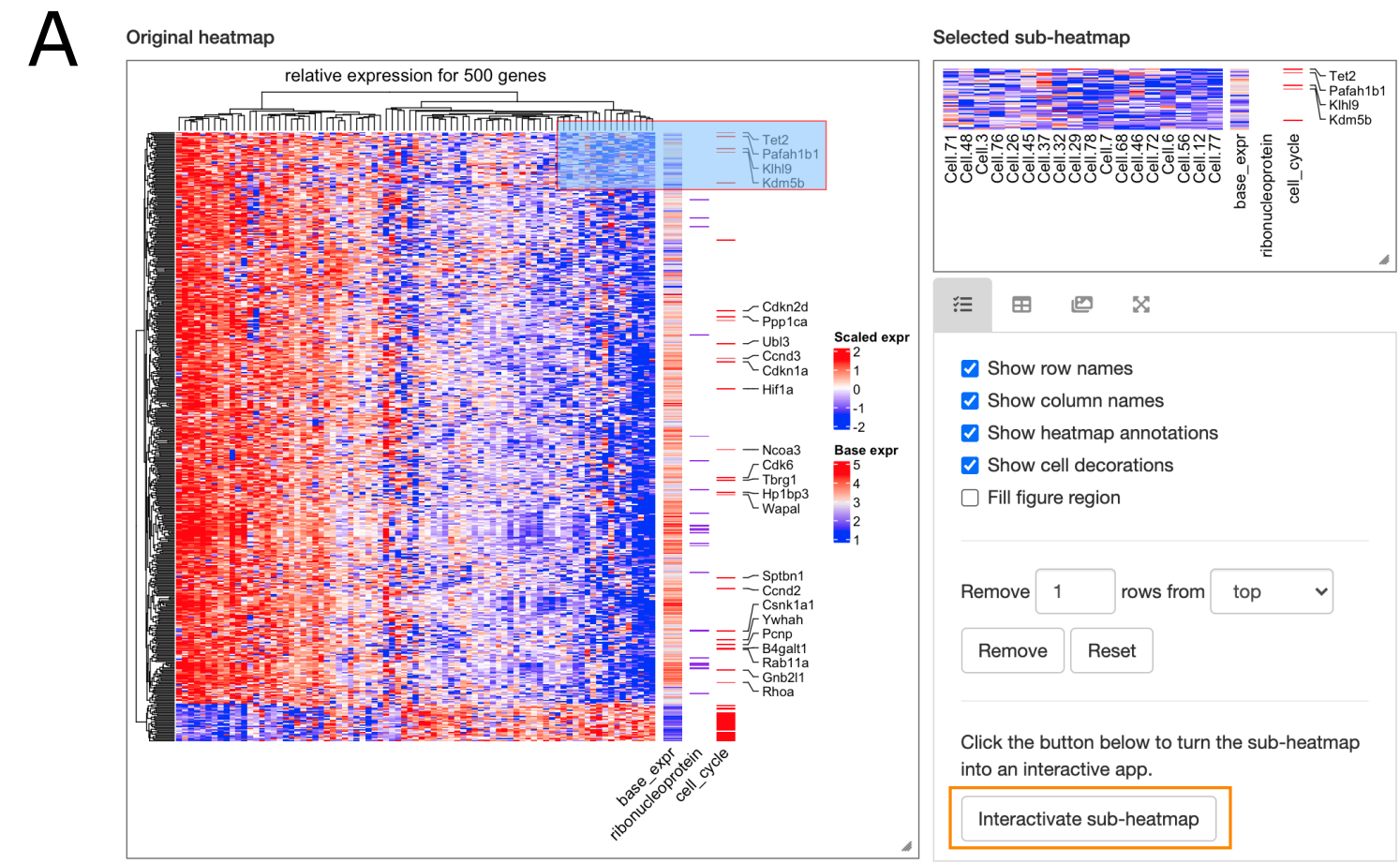

Q 둥 8

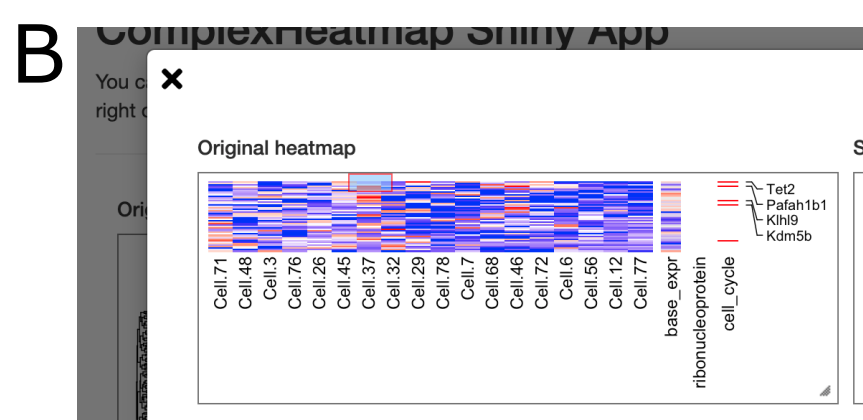

Q 물

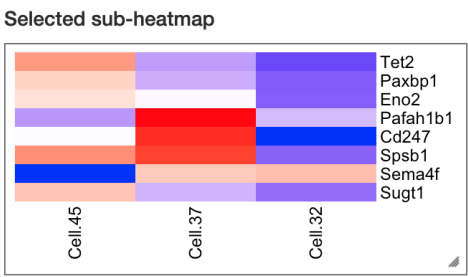

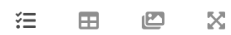

Output

Selected over 1 heatmap with 8 rows and 3 columns. Row and column indices can be obtained by copying following code:

show/hide code

Close widget

Figure 4: Recursively generate interactive heatmaps. The second interactive heatmap (Figure $\mathrm{B}$ ) is generated from the sub-heatmap in the first interactive heatmap by clicking the button "Interactivate sub-heatmap" (highlighted in orange rectangle in Figure A). 
bioRxiv preprint doi: https //doi org/10.1101/2021.03 08.434289; this version posted May 18,2021 . The copyright holder for this preprint (which was not certified by peer review) is the author/funder, who has granted bioRxiv a license to display the preprint in perpetuity. It is made available under aCC-BY-ND 4.0 International license.

A

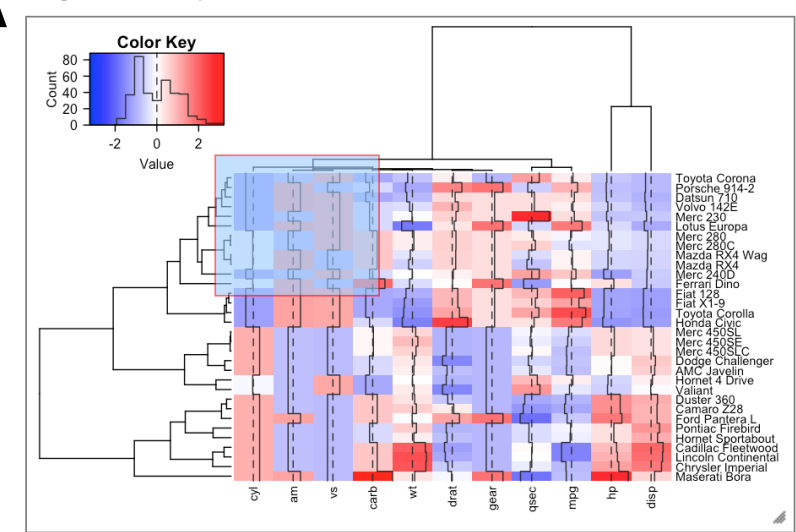

Q 둥 붔

B

Original heatmap

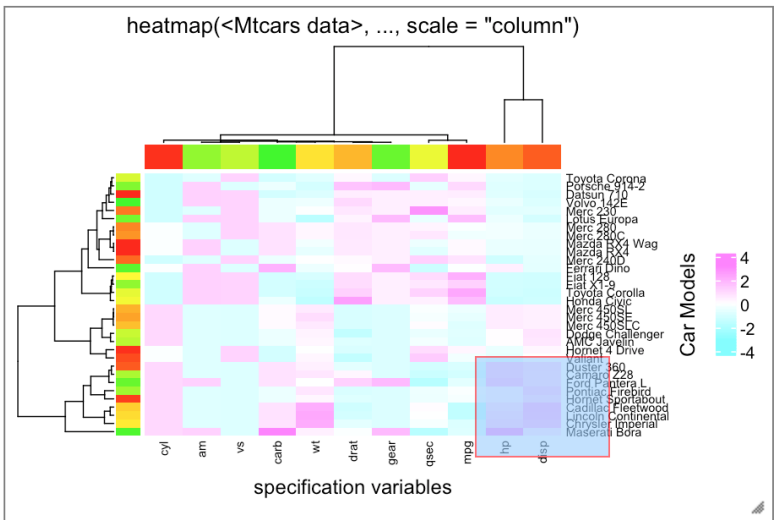

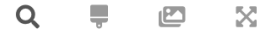

C

Original heatmap

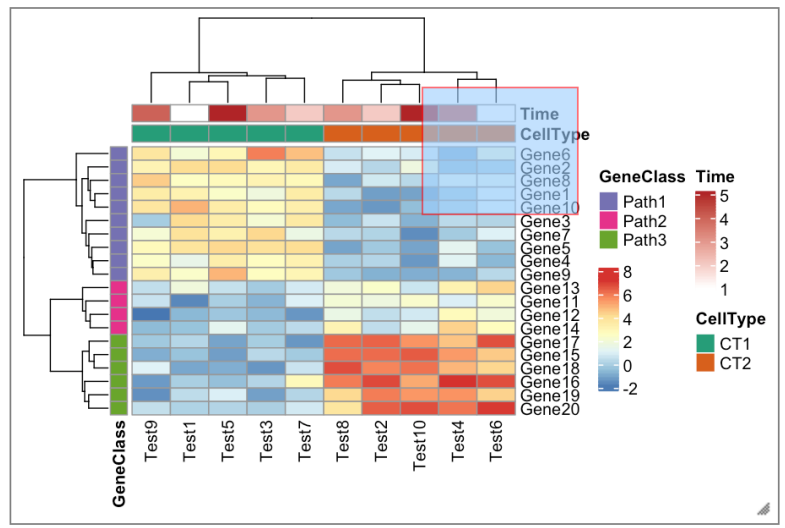

Q 둥 뚤
Selected sub-heatmap

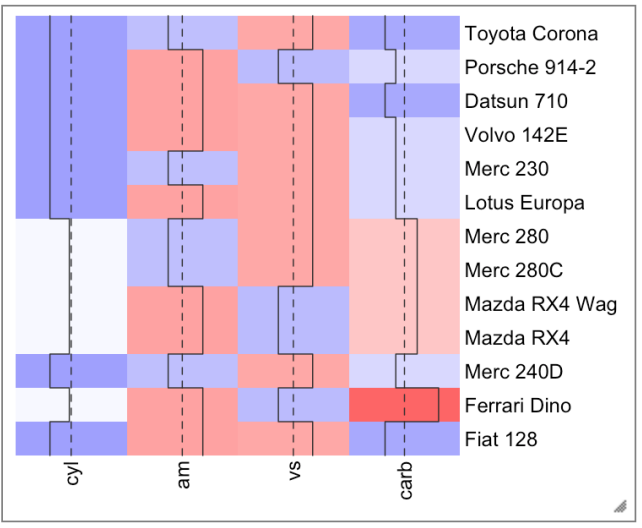

纵田曲跑

Selected sub-heatmap

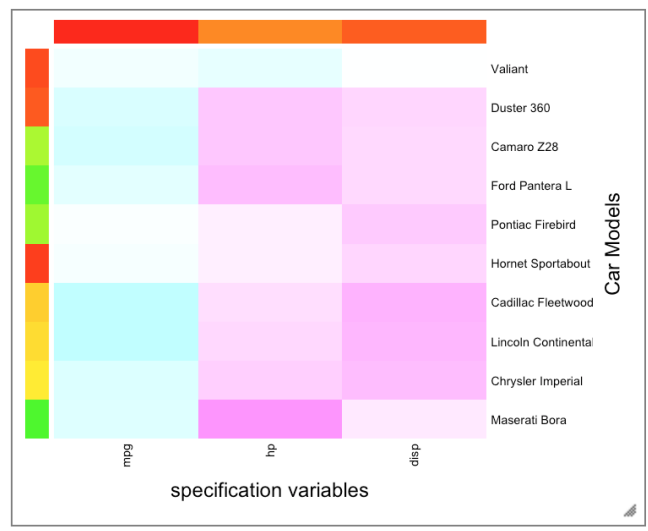

決田曲政

Selected sub-heatmap

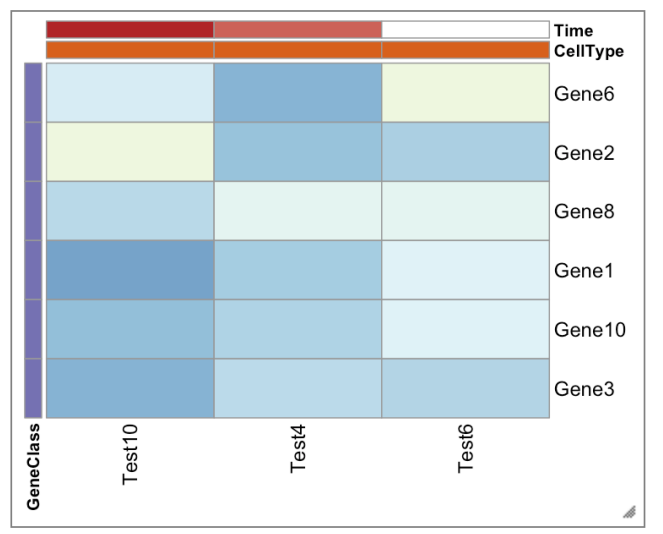

決田串父

Figure 5: Applications on heatmaps generated from other heatmap functions. The three interactive heatmaps are from A) heatmap (), B) heatmap.2() and C) pheatmap(). 
bioRxiv preprint doi: https://doi.org/10.1101/2021.03.08.434289; this version posted May 18, 2021. The copyright holder for this preprint (which was not certified by peer review) is the author/funder, who has granted bioRxiv a license to display the preprint in perpetuity. It is made available under aCC-BY-ND 4.0 International license.

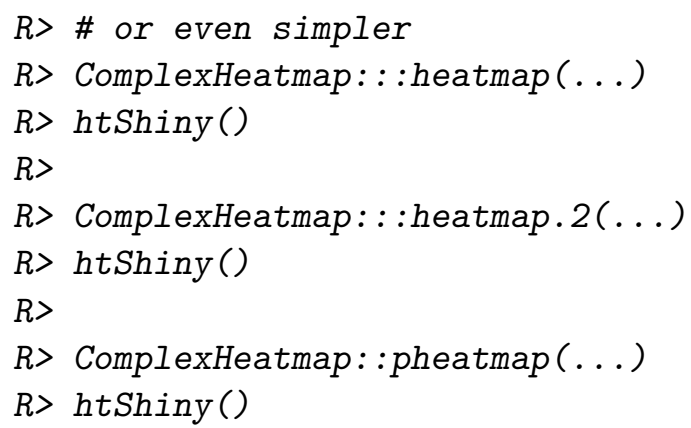

Demonstrations are in Figure 5 and live examples on the three heatmap functions can be obtained from htShinyExample (2.4), htShinyExample (2.5) and htShinyExample (2.6).

\subsection{Applications on other high-level plots}

ComplexHeatmap supports implementing high-level plots as long as the data is represented in a matrix-like structure. The following listed functions all generate Heatmap objects, thus, they can be easily exported to interactive applications.

- ComplexHeatmap: : oncoPrint(): It visualizes various types of genomic alterations for a list of genes in multiple patients and generates a so-called oncoPrint (Gao, Aksoy, Dogrusoz, Dresdner, Gross, Sumer, Sun, Jacobsen, Sinha, Larsson, Cerami, Sander, and Schultz 2013). In the plot, columns are reordered in a special way to highlight the mutual exclusivity of genes that have alterations among different groups of patients. The example of an interactive oncoPrint can be found in Figure 6A and a live example can be run with htShinyExample(2.2).

- ComplexHeatmap: :UpSet(): It implements the UpSet plot (Conway et al. 2017) which is an efficient way for visualizing intersections in a large number of sets. Additionally, UpSet () is able to visualize intersections between multiple lists of genomic intervals. The example of an interactive UpSet plot can be found in Figure $6 \mathrm{~B}$ and a live example can be run with htShinyExample(2.3).

- ComplexHeatmap: :densityHeatmap(): It visualizes a list of distributions by heatmap. The example of an interactive density heatmap can be found in Figure $6 \mathrm{C}$ and a live example can be run with htShinyExample(2.1).

- EnrichedHeatmap: : EnrichedHeatmap(): The package EnrichedHeatmap visualizes the enrichment of certain genomic signals (e.g., DNA methylation) on specific genomic features (e.g., gene TSS) by a special heatmap. EnrichedHeatmap inherits ComplexHeatmap and the constructor function EnrichedHeatmap() returns a Heatmap object, thus, an "enriched heatmap" can be exported to an interactive application as well. One simple example is in Figure 6D and runnable examples can be found in htShinyExample(3.1), htShinyExample(3.2) and htShinyExample(3.3).

Recall ComplexHeatmap makes it easy to combine a list of Heatmap and HeatmapAnnotation objects, thus, heatmaps generated by the previous four functions can associate to additional heatmaps or annotations to generate complex interactive visualizations. An example is in 
bioRxiv preprint doi: https://doi.org/10.1101/2021.03.08.434289. this version posted May 18, 2021. The copyright holder for this preprint (which was not certified by peer review) is the author/funder, who has granted bioRxiv a license to display the preprint in perpetuity. It is made available under aCC-BY-ND 4.0 International license.

Zuguang Gu, Daniel Hübschmann
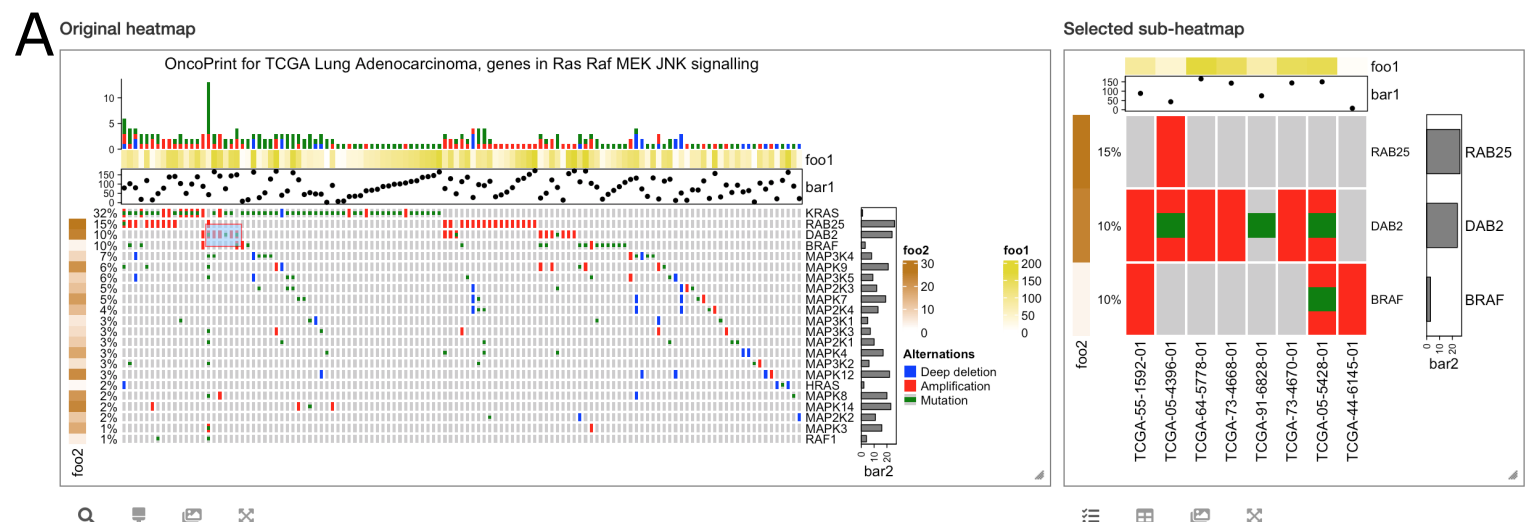

B o.

Q 투 붕

田回

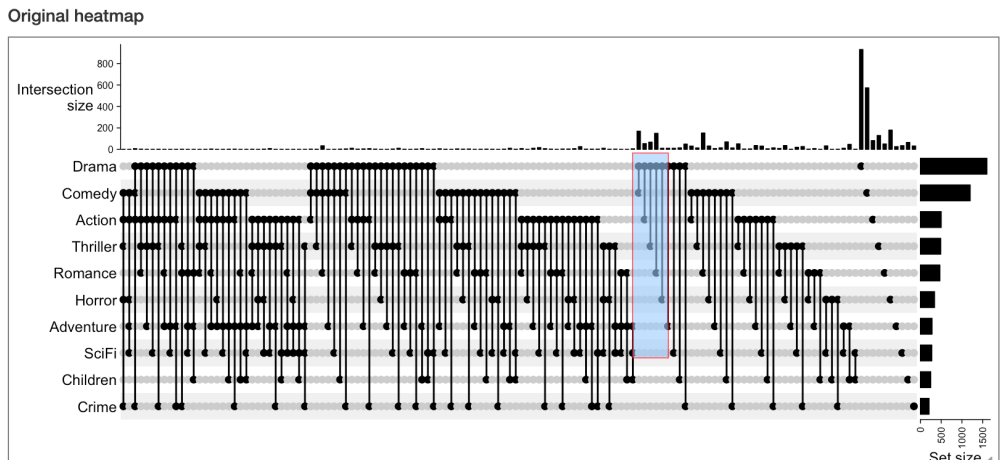

Selected sub-heatmap
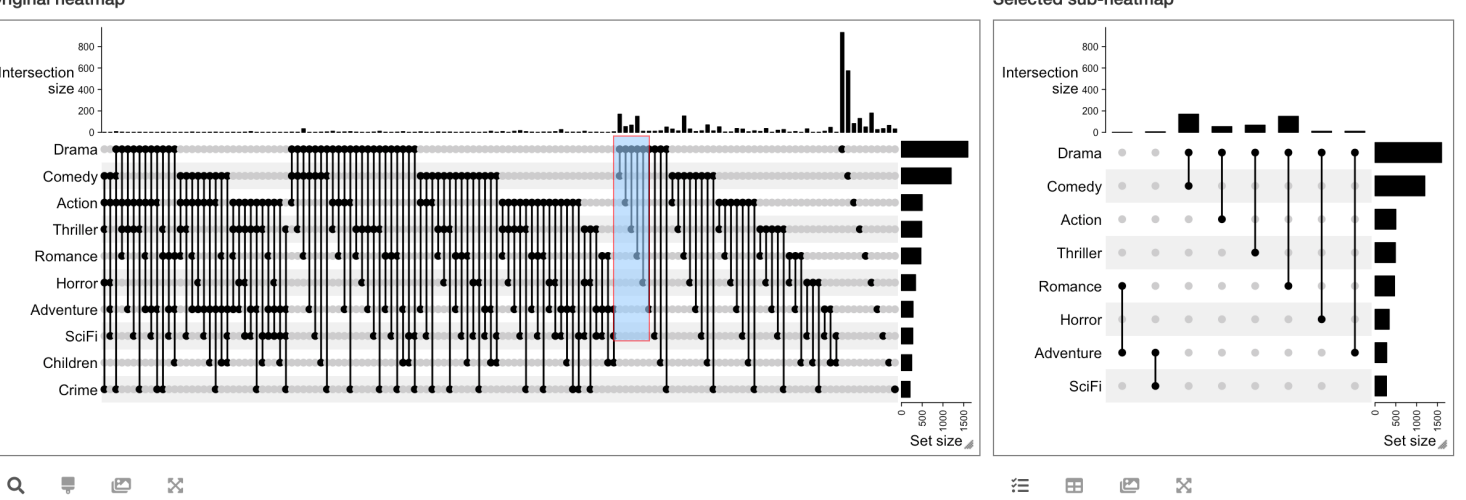

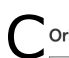

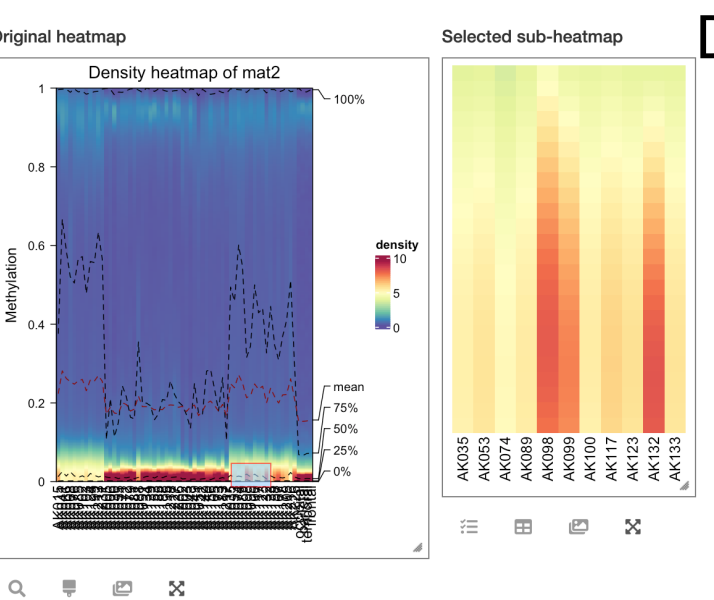

D original heatmap

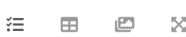

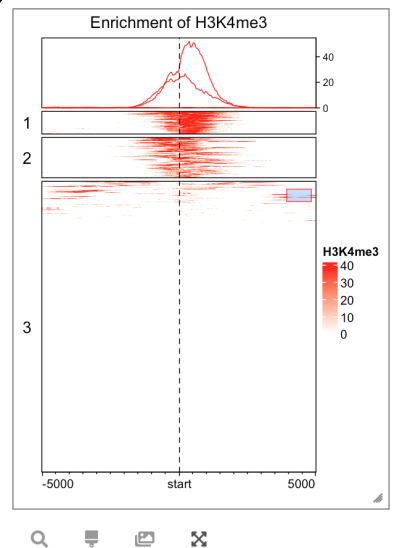

Selected sub-heatmap

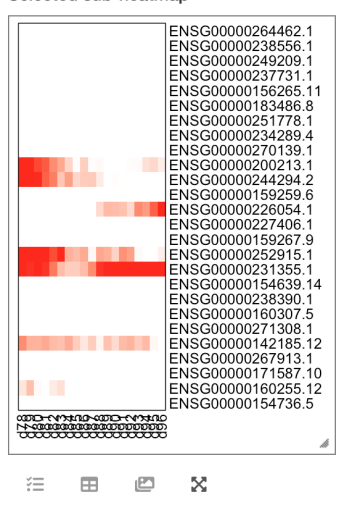

Q 푸

Figure 6: Applications on other high-level plots. A) An interactive oncoPrint. B) An interactive UpSet plot. C) An interactive density heatmap. D) An interactive enriched heatmap. 
htShinyExample (3.2) where the heatmap list includes an enriched heatmap of histone modification on gene TSS, a second enriched heatmap of DNA methylation on gene TSS and a third normal heatmap of gene expression; the heatmap list clearly illustrates the correspondence between three genomic signals and the interactivity of this Shiny application ensures the genes with specific patterns can be easily captured.

As we have demonstrated in Section 3.3, for some other high-level plots generated by functions which internally use ComplexHeatmap while do not directly return Heatmap or HeatmapList objects (e.g., cola: :get_signatures()), users can always first generate heatmap in a certain graphics device (on-screen or off-screen), then execute htShiny() with no heatmap object to obtain the interactive applications (see examples in htShinyExample(1.6) and htShinyExample(1.7)).

\subsection{Interactivate heatmaps indirectly generated by heatmap () , heatmap.2( $)$ and pheatmap ()}

In Section 3.3, we introduced how to export heatmaps indirectly generated from ComplexHeatmap to interactive applications. There is another scenario where heatmaps are indirectly generated by stats: :heatmap(), gplots: :heatmap.2() or pheatmap::pheatmap(), i.e., they are generated by third-party functions which internally use these three heatmap functions. How can we turn these heatmaps into interactive applications? The solution is simple. We just need to go to e.g. pheatmap namespace and replace pheatmap with ComplexHeatmap: : pheatmap right there.

The following example is from package SC3 (Kiselev, Kirschner, Schaub, Andrews, Yiu, Chandra, Natarajan, Reik, Barahona, Green, and Hemberg 2017) where function sc3_plot_expression() draws a heatmap internally using pheatmap: : pheatmap() (This can be found out by checking the source code of sc3_plot_expression()).

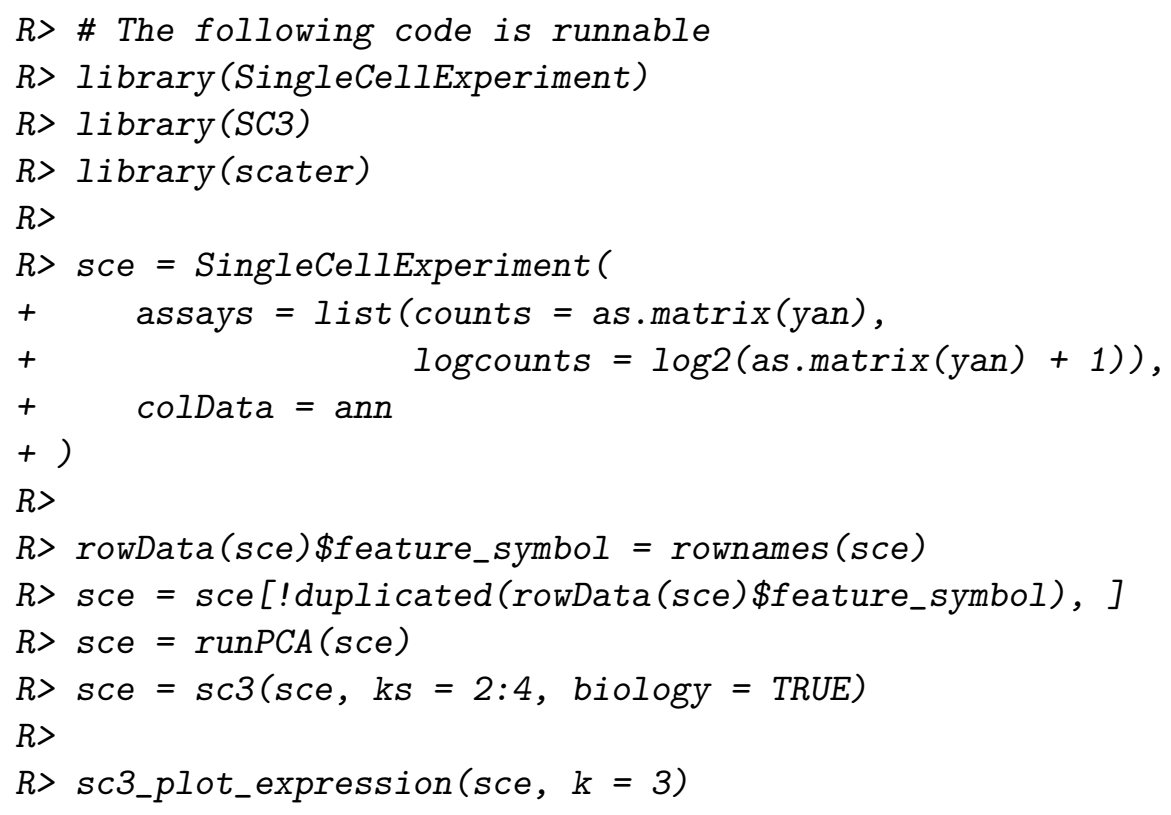

To replace the internal use of pheatmap: :pheatmap() with ComplexHeatmap: :pheatmap(), the function assignInNamespace () can be used to directly change the value of pheatmap 
in pheatmap namespace. After doing that, executing sc3_plot_expression() always uses ComplexHeatmap: :pheatmap () and now it is possible to use htShiny() to export it to an interactive application.

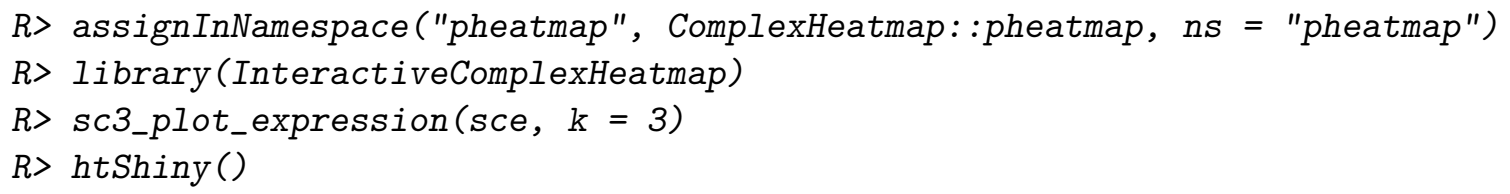

To safely change stats: :heatmap(), gplots: heatmap.2() or pheatmap::pheatmap() to ComplexHeatmap: : :heatmap (), ComplexHeatmap: : :heatmap.2() or ComplexHeatmap: :pheatmap(), it is recommended to execute one of the following commands at the beginning of user's R session.

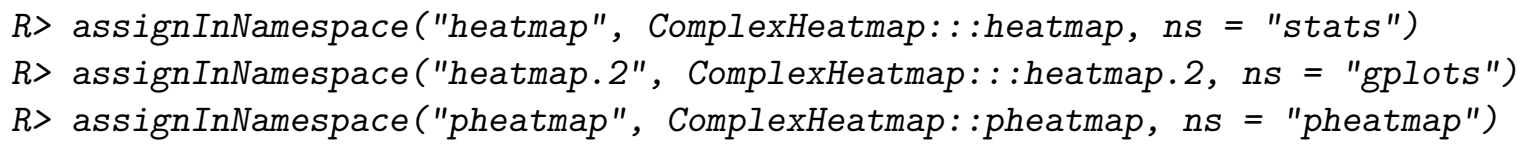

\section{Implementation of interactivity}

Being different from interactive heatmap packages based on JavaScript, e.g., d3heatmap, heatmaply or iheatmapr, InteractiveComplexHeatmap has a special way to capture positions that users clicked on heatmaps and to extract values from corresponding matrices. To demonstrate it, we still use the object ht_list previously generated in Section 3.1 which includes a list of two heatmaps and $k$-means clustering was applied on the numeric heatmap.

InteractiveComplexHeatmap implements two types of interactivity: 1 . on the interactive graphics device, and 2. in the Shiny web application. The interactivity on the interactive graphics device is the basis of the interactivity in the Shiny application, thus, in following sections, we will first introduce how the interactivity is implemented with the interactive graphics device.

\subsection{On the interactive graphics device}

Here the "interactive graphics device" refers to the window for generating plots if $\mathrm{R}$ is directly used in the terminal, or the figure panel in Rstudio IDE.

InteractiveComplexHeatmap first captures physical positions of all heatmap slices, i.e., the distance to the bottom left of the graphics device, by the function htPositionsOnDevice(). Internally, the function goes to the viewport of every heatmap slice and utilizes the function grid: :deviceLoc() to capture positions measured in the graphics device. Before executing htPositionsOnDevice(), the heatmap should be already drawn on the device so that htPositionsOnDevice() can access various viewports.

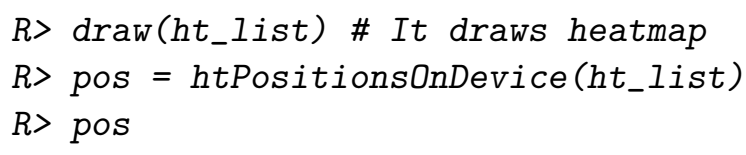




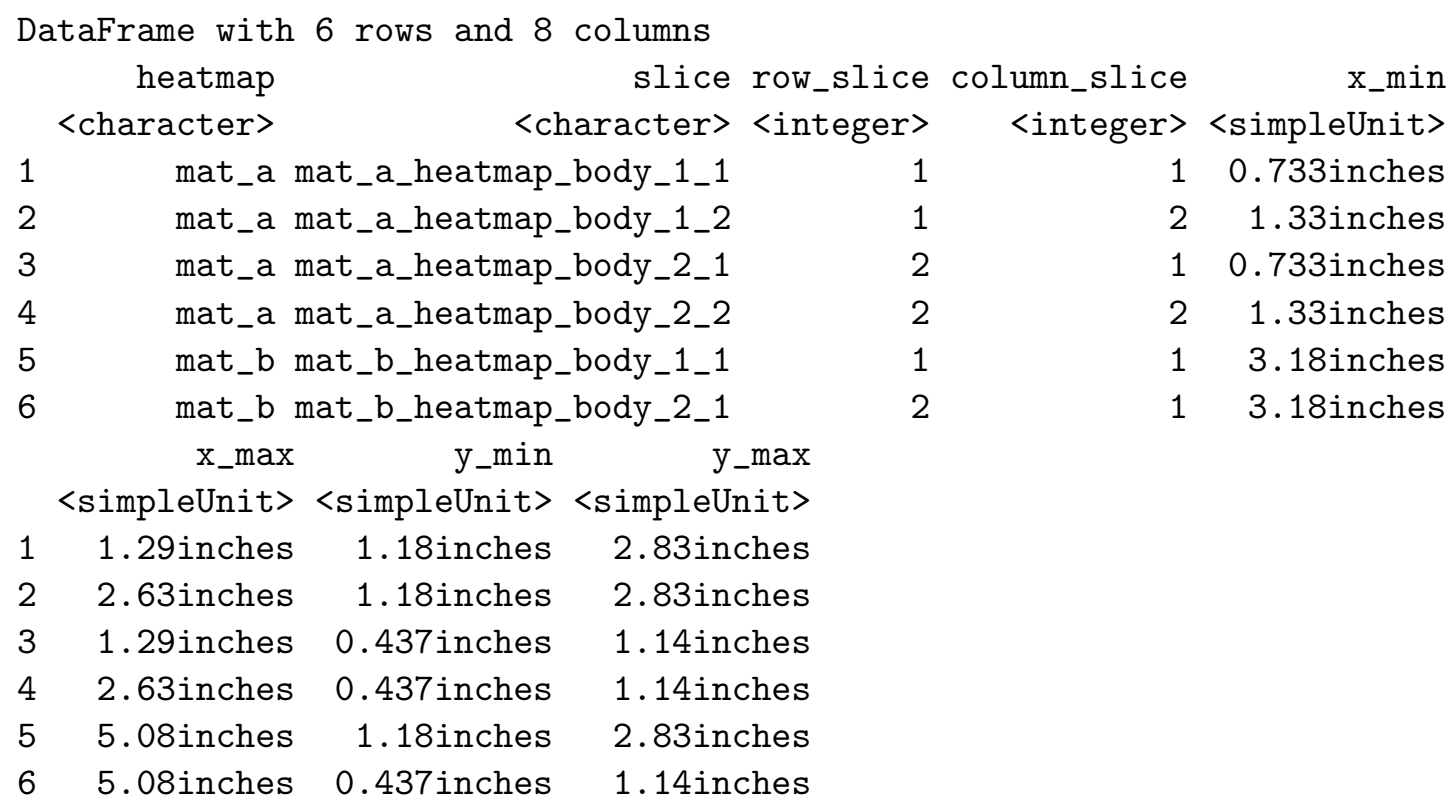

Note, the calculation of positions of heatmap slices relies on the device size. In the previous example code, pos was calculated in a device with 6 inches width and 4 inches height.

The returned object pos is a DataFrame object that contains positions of all heatmap slices. The DataFrame class is defined in package S4Vectors (Pagès, Lawrence, and Aboyoun 2020) and is very similar to a data frame, but it can store more complex data types, such as the simpleUnit vectors generated by grid: :unit(). Figure 7A contains the original heatmap and black rectangles in Figure 7B were drawn based on the positions that were captured.

Next, InteractiveComplexHeatmap captures the physical position of the point that the user clicked on the heatmap. This is simply done by applying function grid: :grid.locator() in the ROOT viewport. Now with knowing positions of heatmap slices and position of the point that the user clicked, it is possible to calculate which row and column in the original matrix that user's click corresponds to.

In Figure 7B, assume blue point with coordinate $(a, b)$ was clicked by the user. The heatmap slice which the user clicked into can be easily identified by comparing the position of the click and the position of every heatmap slice. Assume the heatmap slice where user's click is in has a range of $\left(x_{1}, x_{2}\right)$ on $x$ direction and a range of $\left(y_{1}, y_{2}\right)$ on $y$ direction. There are $n_{r}$ rows $\left(n_{r}=7\right)$ and $n_{c}$ columns $\left(n_{c}=7\right)$ in this heatmap slice and they are marked by dashed lines in Figure 7B. Note all coordinate values $\left(a, b, x_{1}, y_{1}, x_{2}\right.$ and $\left.y_{2}\right)$ are measured as the distances to the bottom left of the graphics device.

In this heatmap slice, the row index $i_{r}$ and column index $i_{c}$ of the cell where the click is in can be calculated as (assume the left bottom corresponds to the index of 1 for both rows and columns):

$$
\begin{aligned}
i_{c} & =\left\lceil\frac{a-x_{1}}{x_{2}-x_{1}} \cdot n_{c}\right\rceil \\
i_{r} & =\left\lceil\frac{b-y_{1}}{y_{2}-y_{1}} \cdot n_{r}\right\rceil
\end{aligned}
$$


bioRxiv preprint doi: https://doi.org/10.1101/2021.03 08.434289; this version posted May 18, 2021. The copyright holder for this preprint (which was not certified by peer review) is the author/funder, who has granted bioRxiv a license to display the preprint in perpetuity. It is made available under aCC-BY-ND 4.0 International license.
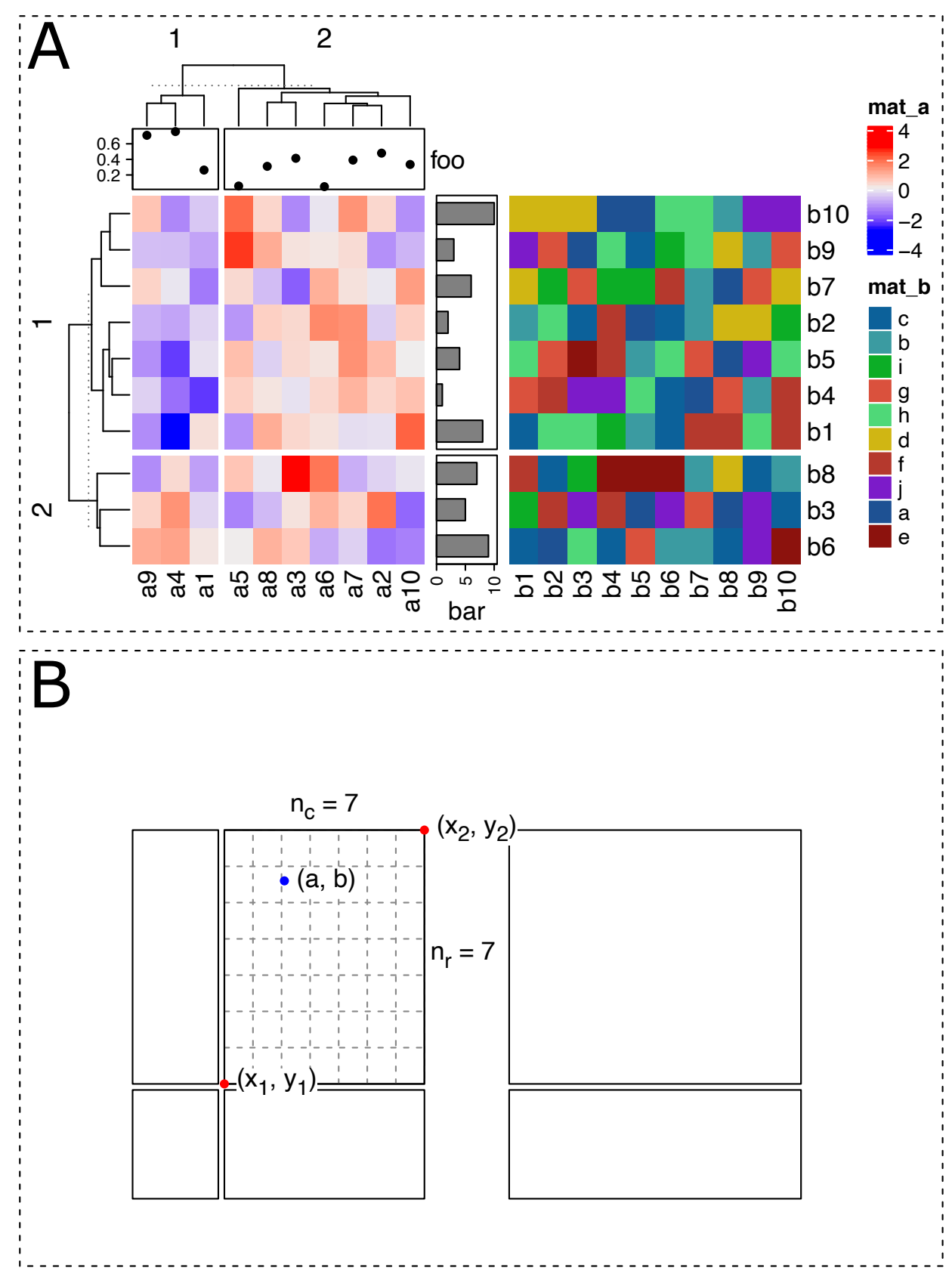

Figure 7: Demonstration of the implementation of interactivity. A) The original heatmap. B) The black rectangles are drawn based on the positions captured for all heatmap slices. Figure A and B were both drawn in a device with 6 inches width and 4 inches height. Dashed rectangles correspond to image borders. 
where the symbol $\lceil x\rceil$ means the ceiling of the numeric value $x$. In ComplexHeatmap, the row with index 1 is always put on the top of the heatmap, then $i_{r}$ should be adjusted to:

$$
i_{r}=n_{r}-\left\lceil\frac{b-y_{1}}{y_{2}-y_{1}} \cdot n_{r}\right\rceil+1
$$

The subset of row and column indices in the original matrix that belongs to the selected heatmap slice is already stored in ht_list object (they can be retrieved by functions row_order() and column_order ()), thus, we can obtain the row and column index in the original matrix that corresponds to user's click easily with $i_{r}$ and $i_{c}$.

Denote the matrix for the complete heatmap (without splitting) as $M$, and denote the subset of row and column indices in the heatmap slice where user's click is in as $o^{\text {row }}$ and $o^{\text {col }}$. Note, $o^{\text {row }}$ and $o^{\text {col }}$ can be reordered due to e.g. clustering. Then row and column indices for the clicked point in $M$, denoted as $j_{r}$ and $j_{c}$, are calculated as follows:

$$
\begin{aligned}
& j_{r}=o_{i_{r}}^{\text {row }} \\
& j_{c}=o_{i_{c}}^{\text {col }}
\end{aligned}
$$

And the corresponding value in $M$ is $M_{j_{r}, j_{c}}$.

InteractiveComplexHeatmap has two functions selectPosition() and selectArea() which allow users to pick single positions or to select areas from heatmap. Under the interactive graphics device, users do not need to run htPositionsOnDevice() explicitly. The positions of heatmaps are automatically calculated, cached and reused if heatmaps are the same and the device has not changed its size. If users change the device size, htPositionsOnDevice() will be automatically re-executed.

Figure 8 demonstrates an example of using selectPosition(). Interactively, selectPosition() asks the user to click one position on the heatmap. The function returns a DataFrame which contains the heatmap name, slice name and row and column index in the corresponding matrix. An example output from selectPosition() is as follows:

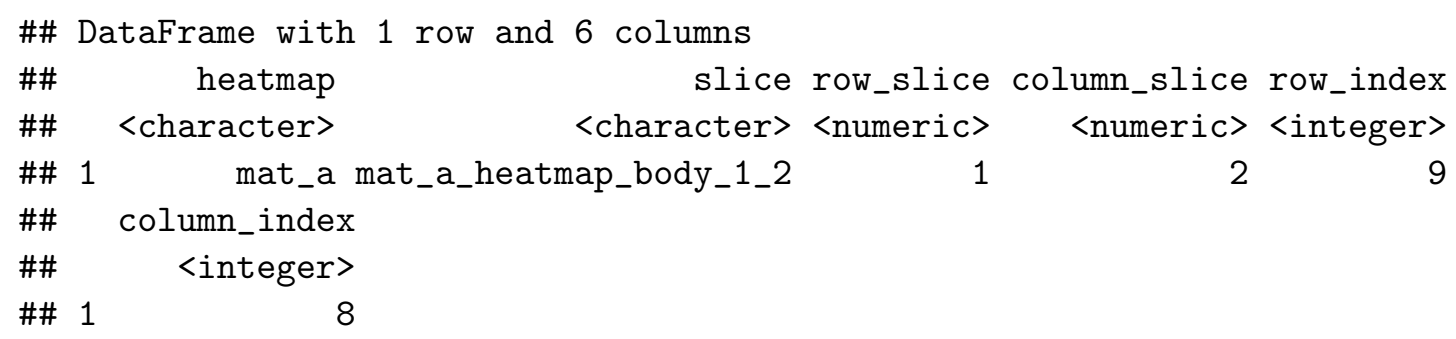

The output means the position that the user clicked is in a heatmap named "mat_a" and in its first row slice and in the second column slice. Assume mat is the matrix for heatmap "mat_a", then the clicked point corresponds to the value mat $[9,8]$.

Similarly, the function selectArea() asks the user to click two positions on the heatmap which define an area. Note since the selected area may overlap over multiple heatmap slices, selectArea() returns a DataFrame with multiple rows. An example output is as follows. 
bioRxiv preprint doi: https://doi org/10.1101/2021.03 08.434289; this version posted May 18, 2021. The copyright holder for this preprint (which was not certified by peer review) is the author/funder, who has granted bioRxiv a license to display the preprint in perpetuity. It is made available under aCC-BY-ND 4.0 International license.
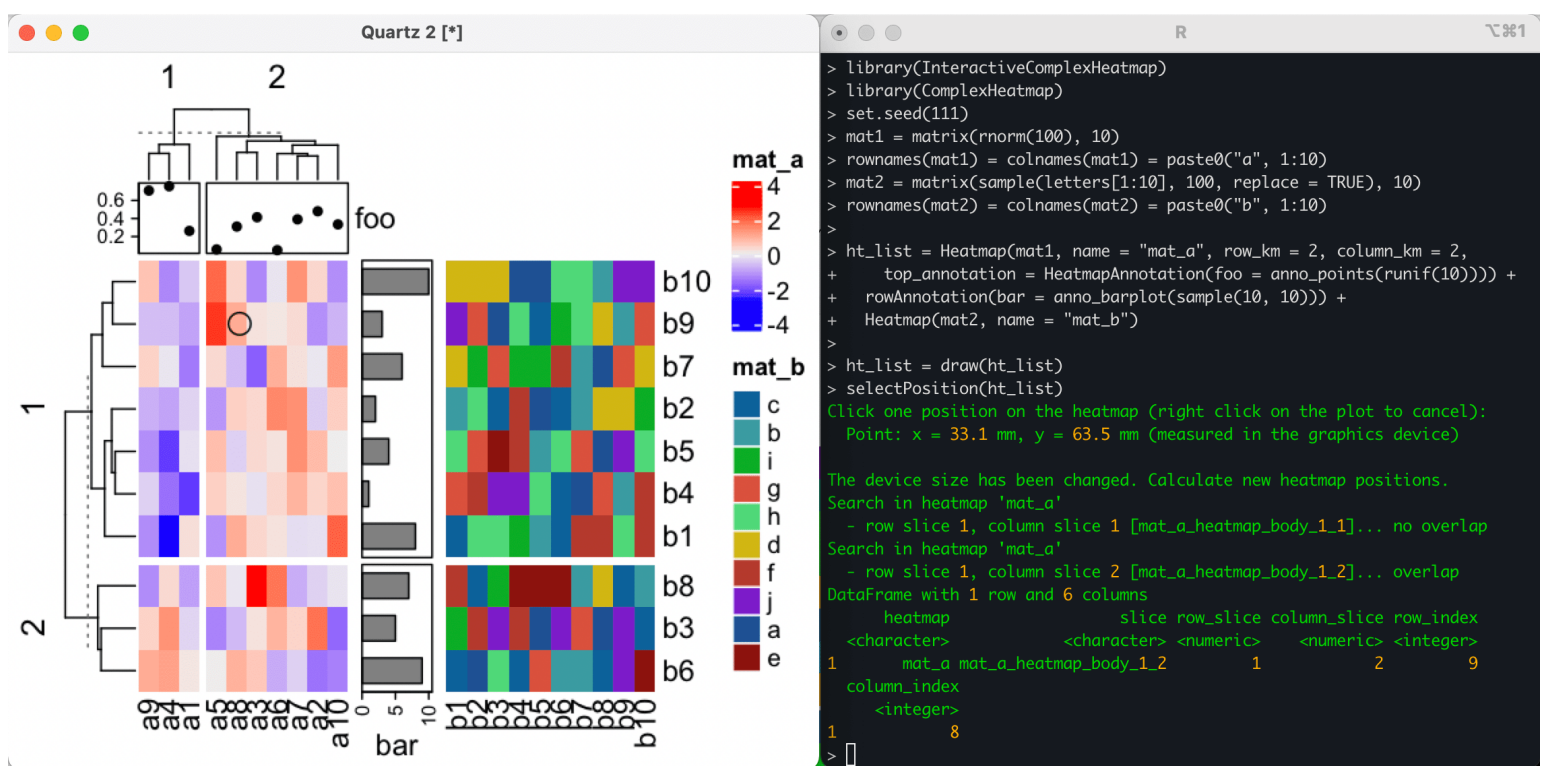

Figure 8: Demonstration of selectPosition(). Circle on the heatmap represents the point clicked by user. On the left is the interactive graphics device and on the right is the $\mathrm{R}$ terminal.

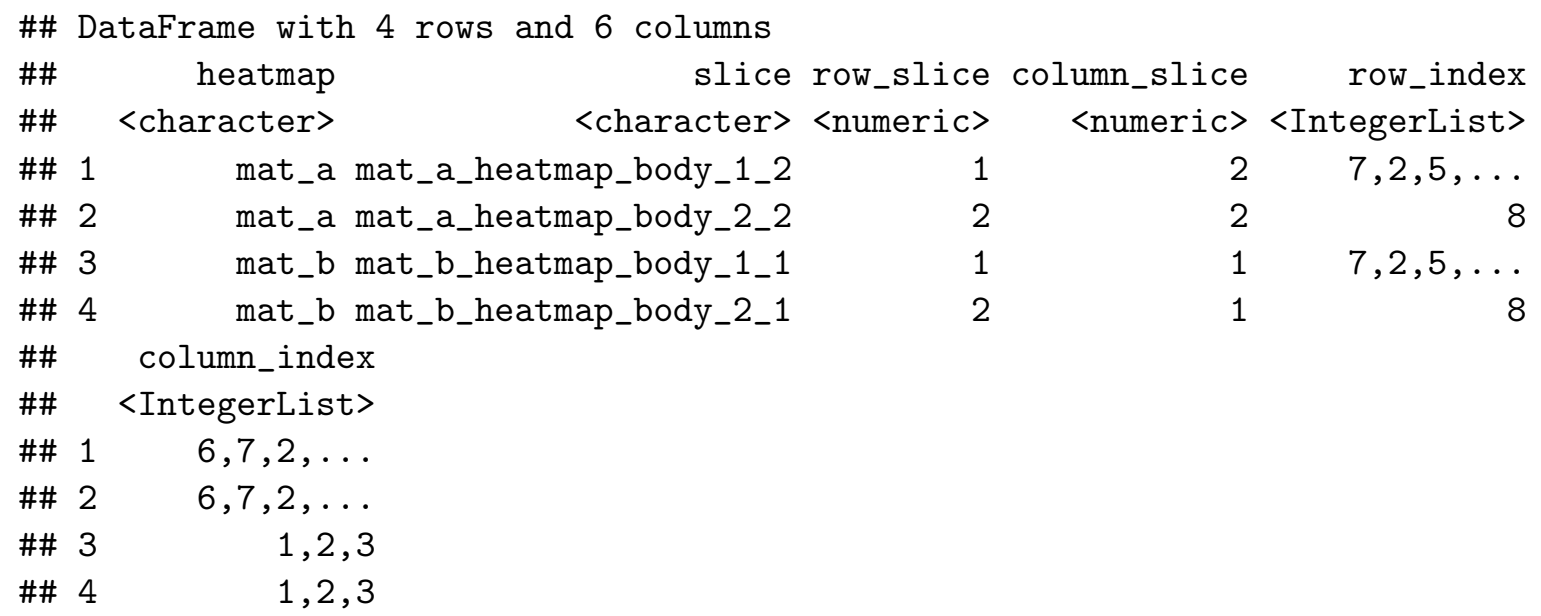

The columns row_index and column_index are stored in a class of IntegerList which is simply a list of integer vectors. To get the row indices in e.g. mat_a_heatmap_body_1_2 (in the first row), user can use either one of the following commands:

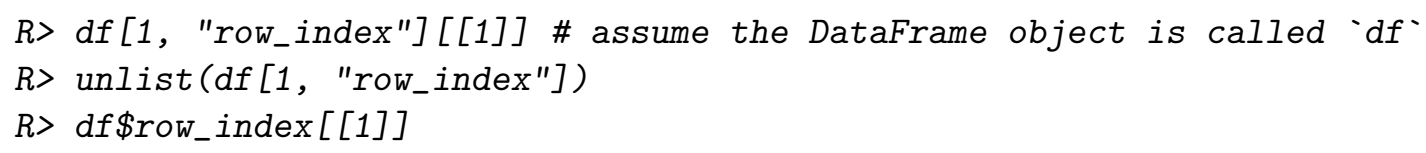

\subsection{On off-screen graphics devices}

It is also possible to use selectPosition() and selectArea() on off-screen graphics devices, such as pdf or png. Now the positions cannot be selected interactively, but instead argument 
pos in selectPosition() or pos1/pos2 in selectArea() need to be specified to simulate clicks. Values for pos, pos1 and pos2 should be unit objects of length two which corresponds to the $x$ and $y$ coordinates relative to the bottom left of the device.

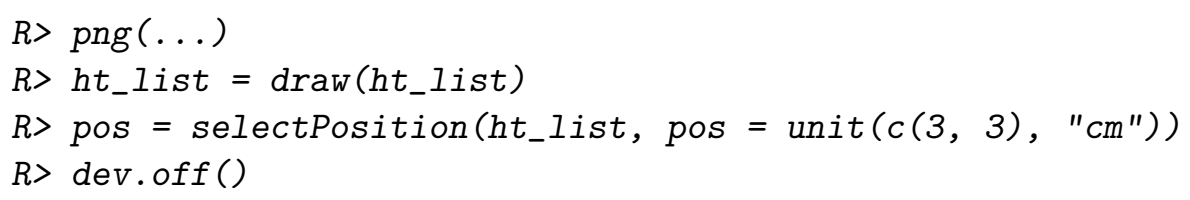

Users do not need to use this functionality directly with an off-screen graphics device, but it is very useful when implementing the interactivity in a Shiny application where the plot is actually generated under an off-screen graphics device.

\subsection{In Shiny web applications}

With the three functions htPositionsOnDevice(), selectPosition() and selectArea(), it is possible to implement Shiny applications for interactively working with heatmaps. Now the question is how does the server side capture the positions that the user clicked on heatmap which is actually on the web page. Luckily, there is a solution. The heatmap is normally generated within a shiny: :plotOutput() and plotOutput() provides two actions that can be applied on heatmaps on the web page: click and brush. Then under the Shiny framework, server can receive the information of the positions as soon as the user performs clicking or brushing on heamtaps. The positions can be later sent to selectPosition() or selectArea() via pos or pos1/pos2 arguments for correctly corresponding to the original matrices. htShiny() is implemented in this way, as well as some other functions for Shiny application development, which will be introduced in the next section.

\section{Shiny web application development}

htShiny() exports heatmaps as stand-alone Shiny web applications. InteractiveComplexHeatmap also provides general solutions for integrating interactive heatmap widgets in general Shiny application development. It allows dynamically generating interactive heatmap widgets according to different configurations on heatmaps, and customizing the output that responds to user's actions on heatmaps.

There are following two main functions for Shiny application development:

- InteractiveComplexHeatmapOutput(),

- makeInteractiveComplexHeatmap().

InteractiveComplexHeatmapOutput() constructs the user interface (UI). As already demonstrated in Section 3.2, the interactive heatmap widget by default contains three components, i.e., the original heatmap, the sub-heatmap and the output that prints information of cells that users selected. The first two heatmap components contain various tools for controlling both heatmaps. makeInteractiveComplexHeatmap() defines a list of responses to user's actions that were performed on heatmaps as well as in the tools. 
bioRxiv preprint doi: https://doi. org/10.1101/2021.03.08.434289. this version posted May 18,2021 . The copyright holder for this preprint (which was not certified by peer review) is the author/funder, who has granted bioRxiv a license to display the preprint in perpetuity. It is made available under aCC-BY-ND 4.0 International license.

\section{My first interactive ComplexHeatmap Shiny app}

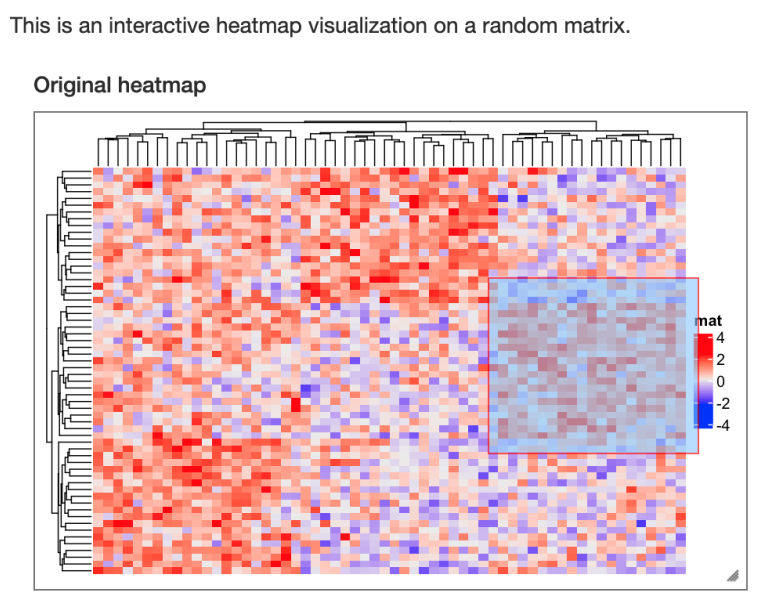

Q 半 X
Selected sub-heatmap

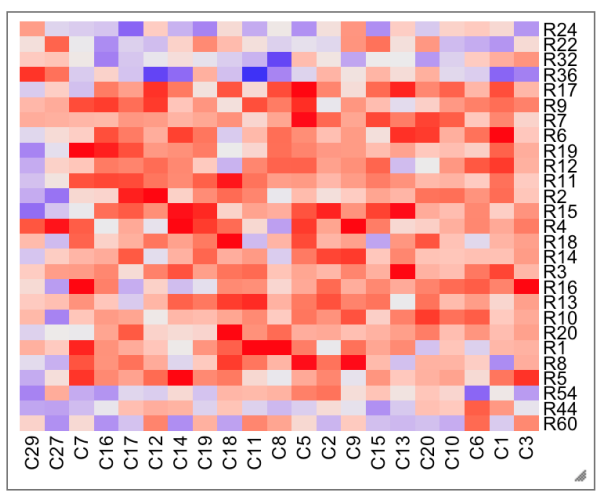

纵田曲边

Output

Selected over 1 heatmap with 27 rows and 21 columns. Row and column indices can be obtained by copying following code:

show/hide code

Figure 9: A simple Shiny application directly from ui and server.

Note htShiny() is simply a wrapper function on these two functions, thus, most of the functionalities introduced in this section also work for htShiny().

\subsection{With one interactive heatmap widget}

Creating a Shiny application that only contains one interactive heatmap widget is rather simple. Following is an example that can be directly copied and pasted to an $\mathrm{R}$ session (Figure 9).

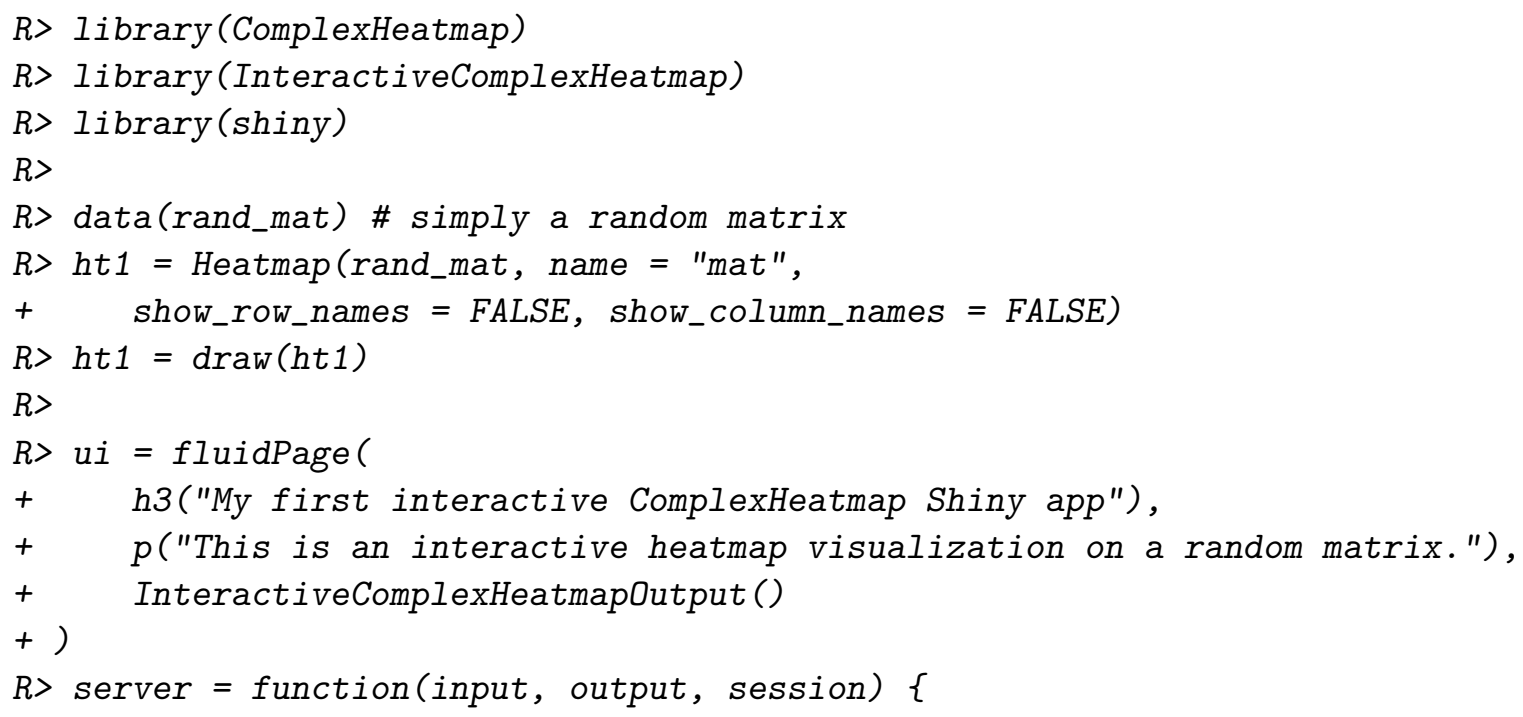




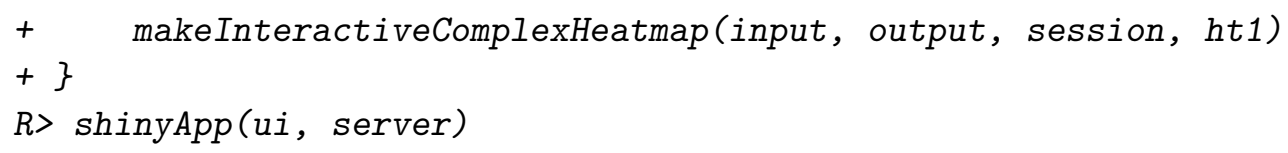

\subsection{With multiple interactive heatmap widgets}

Multiple interactive heatmap widgets can be integrated into one single Shiny application. Now a "heatmap ID" must be assigned to each widget, so that makeInteractiveComplexHeatmap () can find the correct heatmap to respond. If there is only one heatmap widget in the Shiny application, such as in Section 5.1, the heatmap ID is internally generated if it is not specified and the UI is automatically linked to the server in the two functions. In the following example, we create a second widget which visualizes a random character matrix (Figure 10). The two interactive heatmap widgets are independent in the application. See htShinyExample(1.8) and htShinyExample(5.1) for live examples.

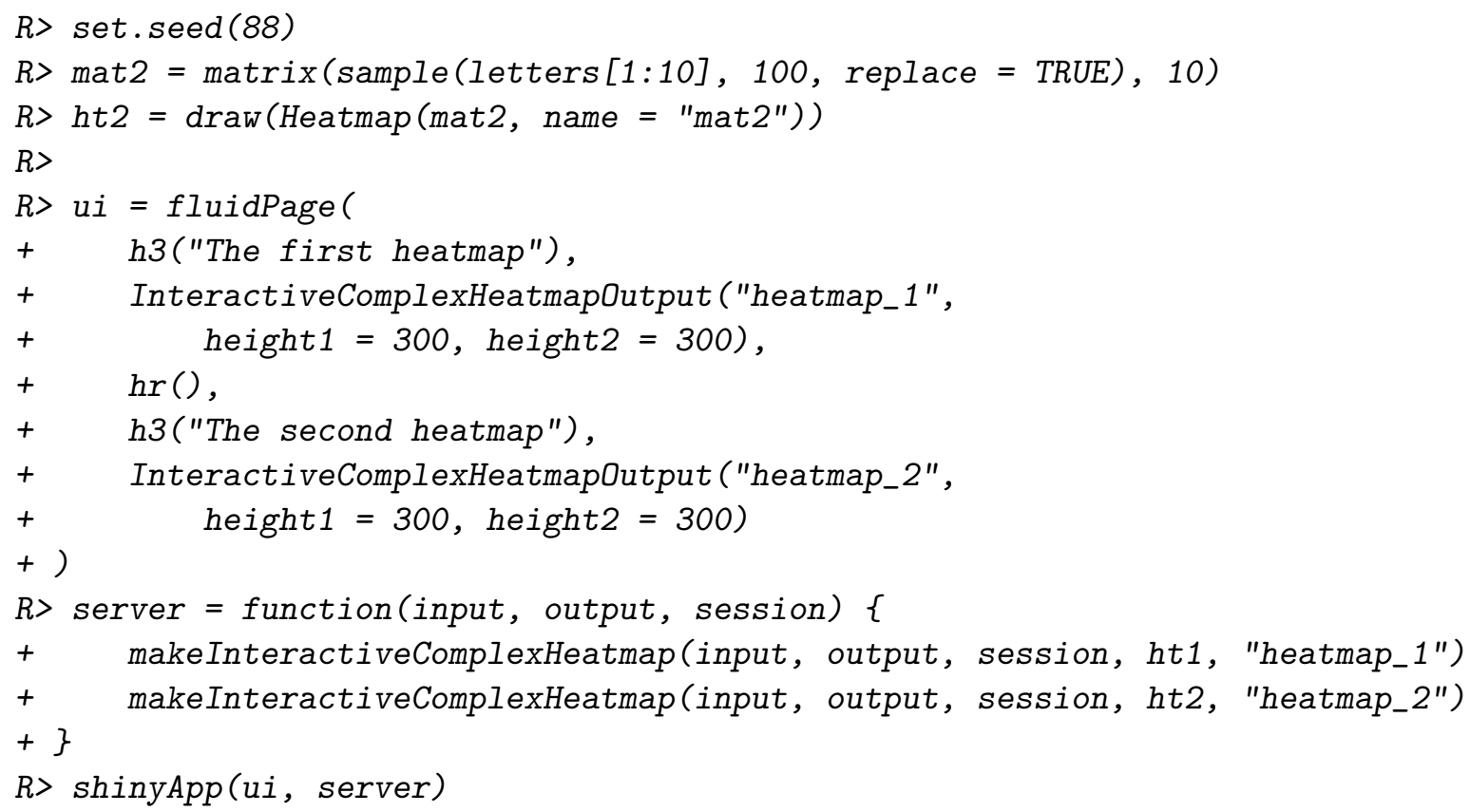

\subsection{Customize the widgets}

The original heatmap and sub-heatmap components can be resized by dragging from bottom right of the two boxes, but still, InteractiveComplexHeatmapOutput() provides arguments of width1, height1, width2 and height2 to precisely control the initial sizes of the two components. They can be manually set to make sure the heatmaps are well visualized and aligned when the application is initialized.

\section{The layout}

The layout of the three components is controlled by argument layout in InteractiveComplexHeatmapOutput(). It supports following values: 
bioRxiv preprint doi: https://doi.org/10.1101/2021.03.08 434289- this version posted May 18,2021 . The copyright holder for this preprint (which was not certified by peer review) is the author/funder, who has granted bioRxiv a license to display the preprint in perpetuity. It is made available under aCC-BY-ND 4.0 International license.

\section{The first heatmap}

Original heatmap

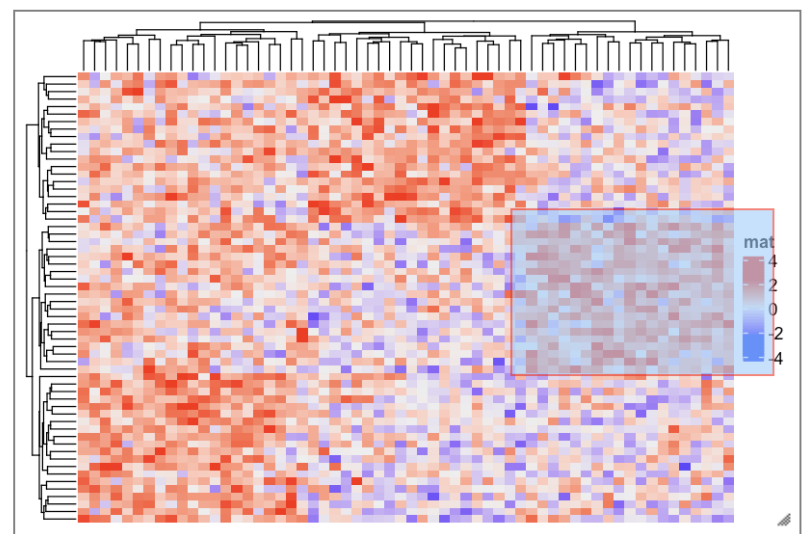

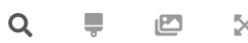

Selected sub-heatmap

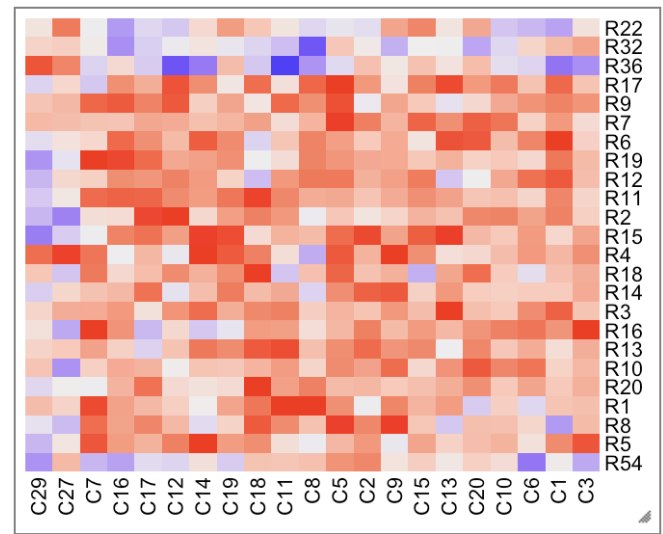

纵田曲要

Output

Selected over 1 heatmap with 24 rows and 21 columns. Row and column indices can be obtained by copying following code:

show/hide code

\section{The second heatmap}

Original heatmap

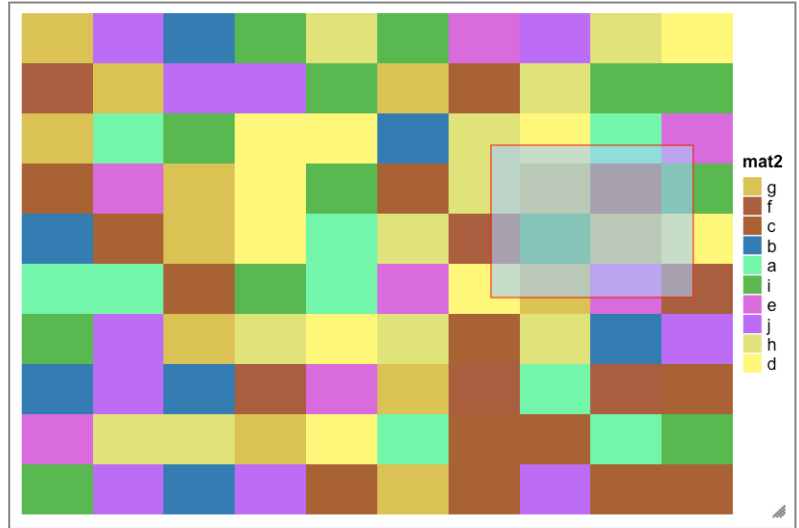

Q 문 만
Selected sub-heatmap

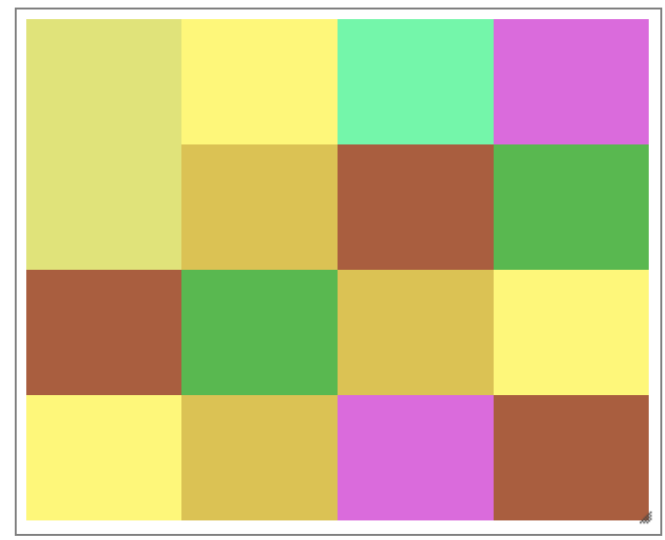

決田曲趾

Output

Selected over 1 heatmap with 4 rows and 4 columns. Row and column indices can be obtained by copying following code:

show/hide code

Figure 10: A Shiny application with two interactive heatmap widgets. 
- "(1-2)|3": Original heatmap and sub-heatmap are in the same row, and output is in the second row. This is the default layout.

- "1| (2-3)": Original heatmap is in a single row, while sub-heatmap and output are in the second row.

- "1-2-3": All three components are in the same row.

- "1|2|3": Each component is in a single row.

- "1-(2|3)": Original heatmap is in a single column. Sub-heatmap and output are vertically aligned and are in the second column. An example can be found with htShinyExample (4.1).

Note the values for layout are in a special format to help to understand the layout, where the three code 1, 2 and 3 correspond to original heatmap, sub-heatmap and output respectively, symbol "-" corresponds to horizontal alignment and " |" corresponds to vertical alignment. With different layouts, different default values are assigned to widths and heights of the three components to make sure they are well aligned.

\section{Action on single heatmap cells}

By default, to get the information of a single cell in the heatmap, a "click" action is used. In InteractiveComplexHeatmapOutput(), the action can also be set to "hover" or "dblclick", then hovering or double clicking on heatmap will trigger the response on the sever side. The example in htShinyExample(1.9) demonstrates usages of these three actions.

\section{Which action to respond}

The argument response in InteractiveComplexHeatmapOutput () can be set as a vector with values in "click", "hover", "dblclick", "brush" and "brush-output" to only respond to one or multiple events on heatmap. E.g., if response is only set to "click", there will be no response for the brush event in the interactive heatmap, also the sub-heatmap component is removed from the application.

Brushing on heatmap by default triggers two responses, one in the sub-heatmap and one in the output. If "brush-output" is included in response instead of "brush", there will only be response in the output component, while the sub-heatmap component is removed from the application.

\section{Separately specify the three UI components}

InteractiveComplexHeatmapOutput() contains all three UI components. Nevertheless, the three components can be separately specified by three individual functions: originalHeatmapOutput(), subHeatmapOutput() and HeatmapInfoOutput(). This provides flexibility for the UI arrangement, e.g., to integrate with package shinydashboard (Chang and Borges Ribeiro 2018) where each UI component is wrapped within an individual box. An example is as follows (Figure 11).

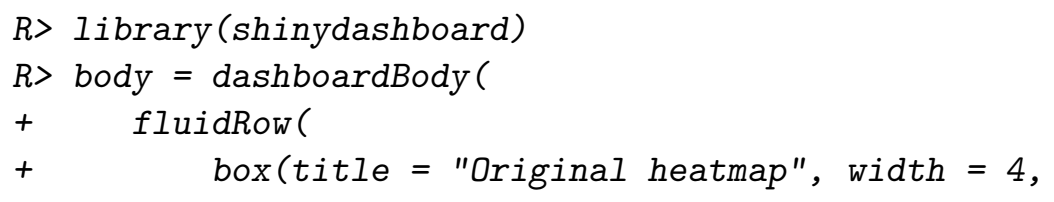




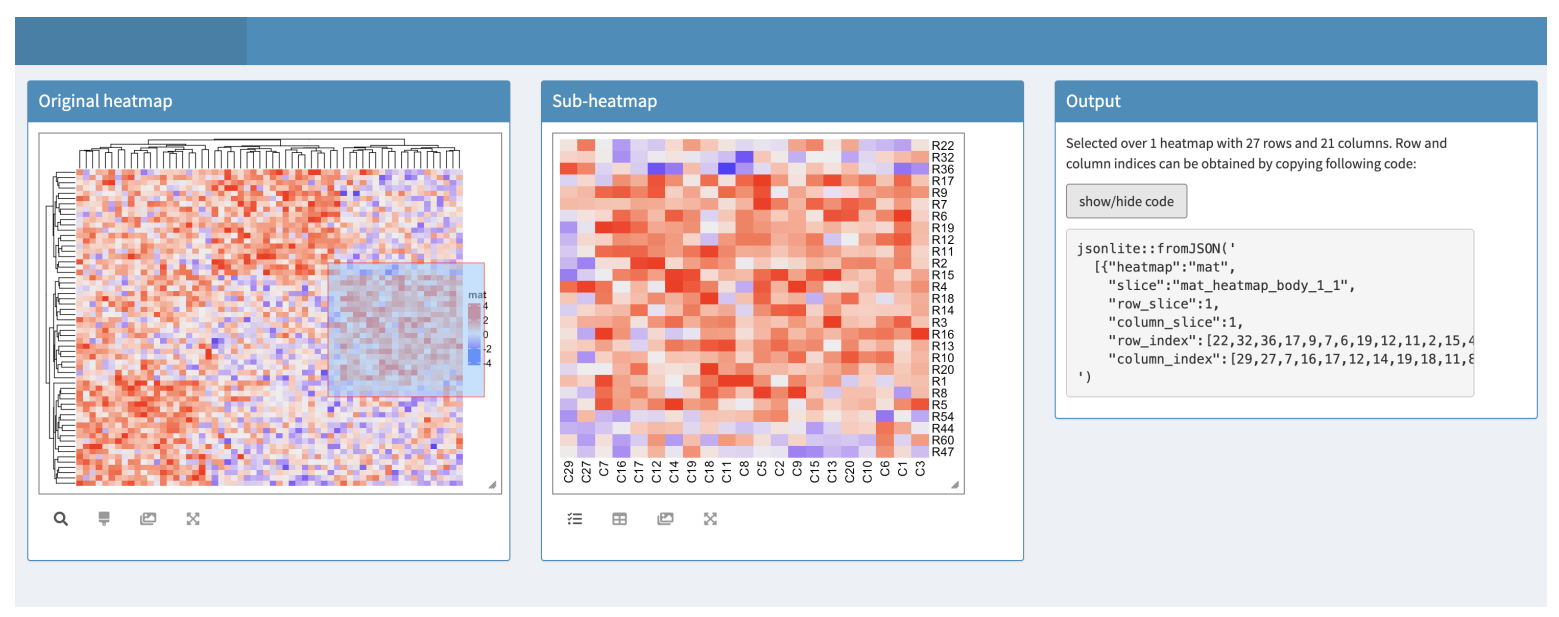

Figure 11: Separately specify the three UI components and integrate with shinydashboard.

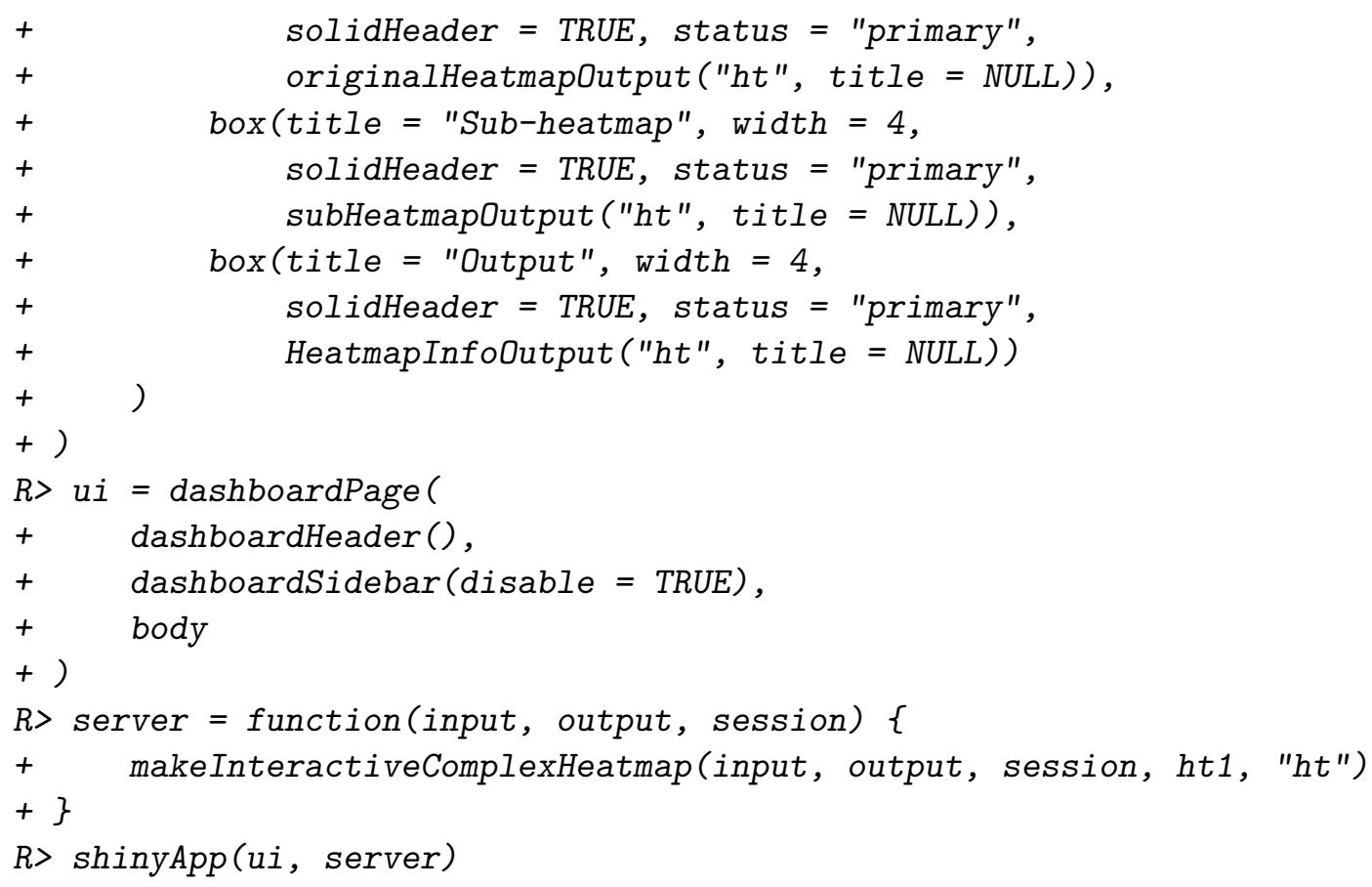

Please note, since now the three components are generated independently, to correctly connect the three components as well as the server, the heatmap ID must be explicitly specified in all functions. More examples on integrating with shinydashboard can be found in htShinyExample(10.1) to htShinyExample(10.5).

\subsection{Work with $\mathrm{R}$ Markdown documents}

It is straightforward to integrate InteractiveComplexHeatmap in an interactive R Markdown document, just in the same way as integrating normal Shiny applications. Examples can be 
found in htShinyExample(7.1) and htShinyExample(7.2).

\subsection{Self-define the output}

Both clicking and brushing on a heatmap trigger an output associated with the heatmap. The output gives information of rows and columns selected by users. In InteractiveComplexHeatmap, the response for the two actions can be self-defined, which makes it possible to build an interactive application with complex responses.

There are two arguments: click_action and brush_action in makeInteractiveComplexHeatmap () which accept self-defined functions to define how to respond after the heatmap was clicked or brushed. The two functions should accept two arguments, the first is a DataFrame object which contains the information of which rows and columns are selected by the user, and the second argument should always be output which is used in the Shiny server function. To use click_action or brush_action, a htmlOutput (or other similar *Output) should be first set up in the UI, then the Shiny application knows where to update the output. The output UI can replace the default output by directly assigning to argument output_ui in InteractiveComplexHeatmapOutput().

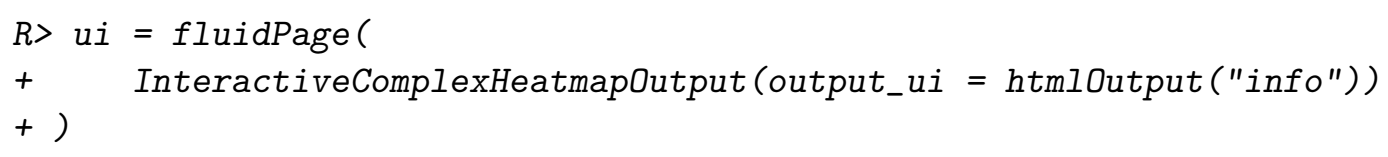

Or to create a new output UI independent to the interactive heatmap widget.

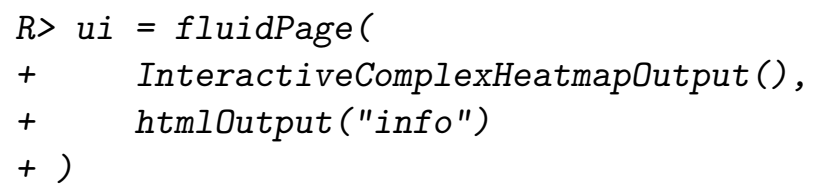

click_action or brush_action is normally defined as follows (assume the ID set in htmlOutput() is "info"):

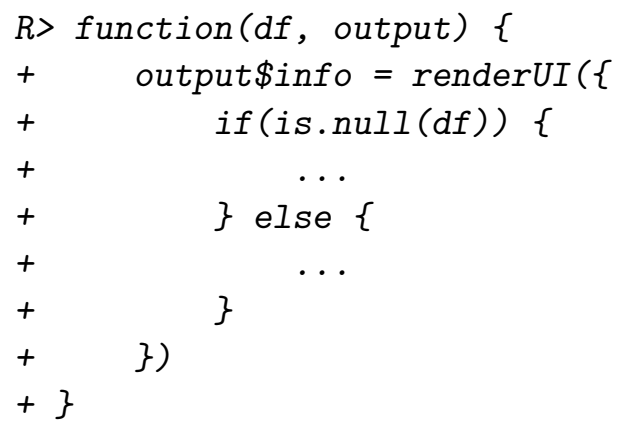

If users didn't click or brush inside the heatmap body (e.g., only clicked in the dendrogram), $\mathrm{df}$ that is passed to the functions will be NULL. A sanity check might be performed here to make specific response when heatmap cell was not selected.

The format of df is slightly different between click and brush. If it is a click action, df has the same format as the returned object of selectPosition() (introduced in Section 4), which looks like the following chunk. It always has one row if the user clicked into the heatmap. 


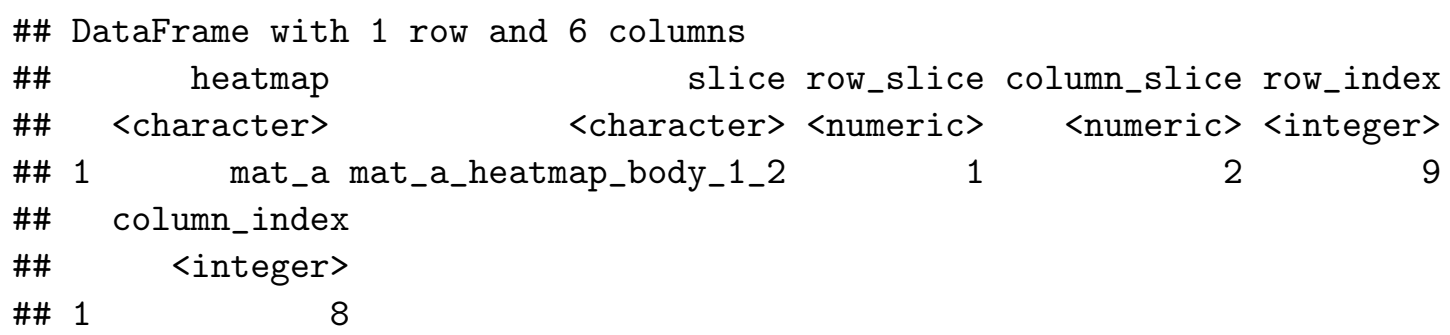

If it is a brush action, $d f$ has the same format as the returned object of selectArea() (introduced in Section 4), which looks like the following chunk. Each line contains row and column indices of the selected sub-matrix in a specific heatmap slice of a specific heatmap.

\begin{tabular}{|c|c|c|c|c|c|}
\hline \#\# & & heatmap & row_slice & column_slice & row_index \\
\hline \#\# & & <character> & $\langle$ numeric $>$ & $\langle$ numeric $>$ & <IntegerList> \\
\hline \#\# & 1 & mat_a mat_a_heatmap_body_1_2 & 1 & 2 & $7,2,5, \ldots$ \\
\hline \#\# & 2 & mat_a mat_a_heatmap_body_2_2 & 2 & 2 & 8 \\
\hline \#\# & 3 & mat_b mat_b_heatmap_body_1_1 & 1 & 1 & $7,2,5, \ldots$ \\
\hline \#\# & 4 & mat_b mat_b_heatmap_body_2_1 & 2 & 1 & 8 \\
\hline \#\# & & column_index & & & \\
\hline \#\# & & <IntegerList> & & & \\
\hline \#\# & 1 & $6,7,2, \ldots$ & & & \\
\hline \#\# & 2 & $6,7,2, \ldots$ & & & \\
\hline \#\# & 3 & $1,2,3$ & & & \\
\hline \#\# & 4 & $1,2,3$ & & & \\
\hline
\end{tabular}

Note as demonstrated above, after a brush action, values in column row_index and column_index might be duplicated due to the fact that the selected heatmap slices are in the same row or column. E.g., in the previous example, the first and the third rows correspond to the selection in the first row slice, but in two different column slices, thus they have the same values for row_index. To safely get row indices and column indices of the selected heatmap, users might need to perform:

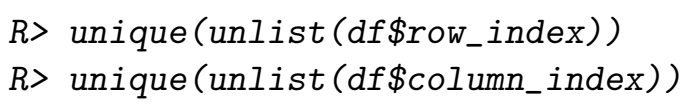

To receive more information from the Shiny application, the self-defined functions for click_action and brush_action can also accept four arguments where two additional variables input and session are passed from server function:

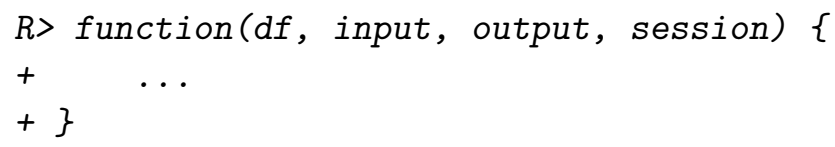

If argument action in InteractiveComplexHeatmapOutput() is set to "hover" or "dblclick", the argument in makeInteractiveComplexHeatmap () is hover_action or dblclick_action correspondingly. Their usages are the same as click_action. 
Original heatmap

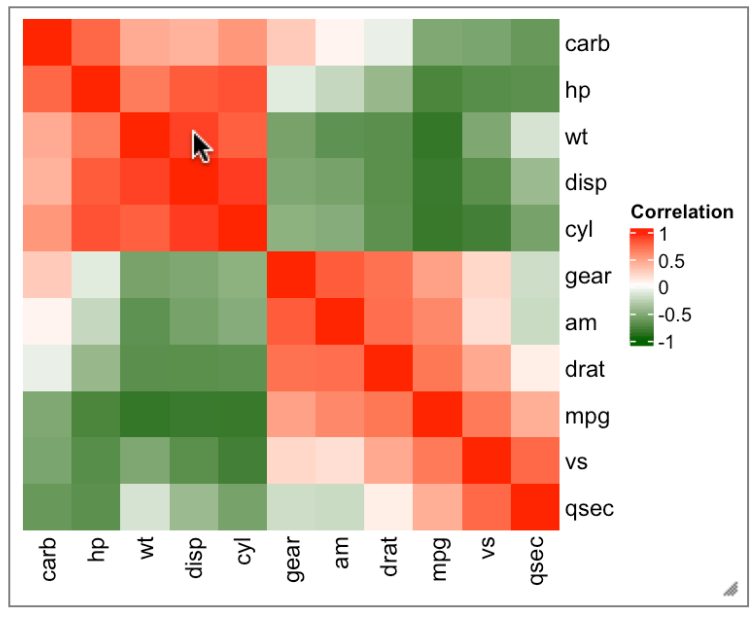

Q 둥 중
Correlation $\mathbf{=} \mathbf{0 . 8 8 8}$

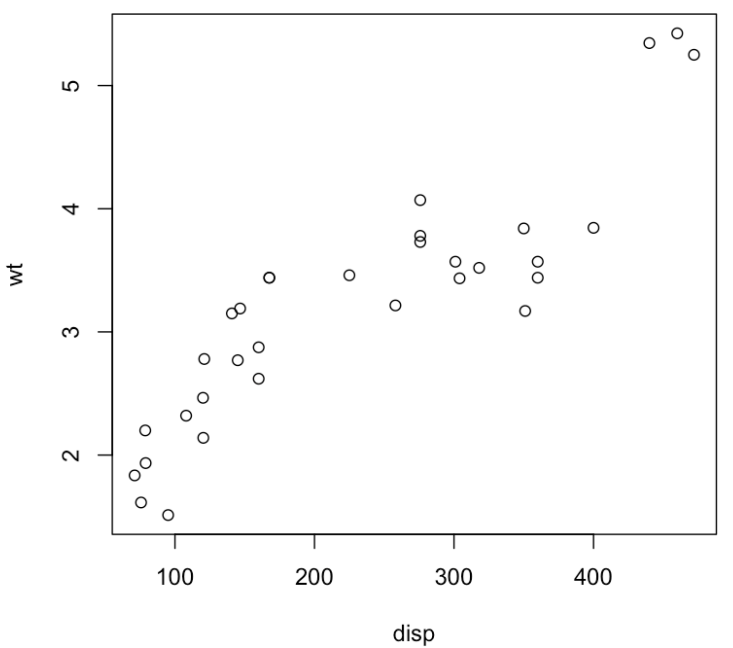

Figure 12: A demonstration of self-defining the output. The heatmap visualizes a correlation matrix based on mtcars dataset. Clicking on a cell draws a scatter plot of the two corresponding variables.

We demonstrate how to self-define click_action in the next example which visualizes a correlation matrix based on mtcars dataset. In the UI, the default output is replaced with a new plotOutput. On the server side, a self-defined click_action is defined to draw a scatter plot of the two corresponding variables that were selected from heatmap (Figure 12). Note in the example response $=$ "click" is set to simplify Figure 12 so that the sub-heatmap component is not included in the application. The live version of this example can be found in htShinyExample(5.5).

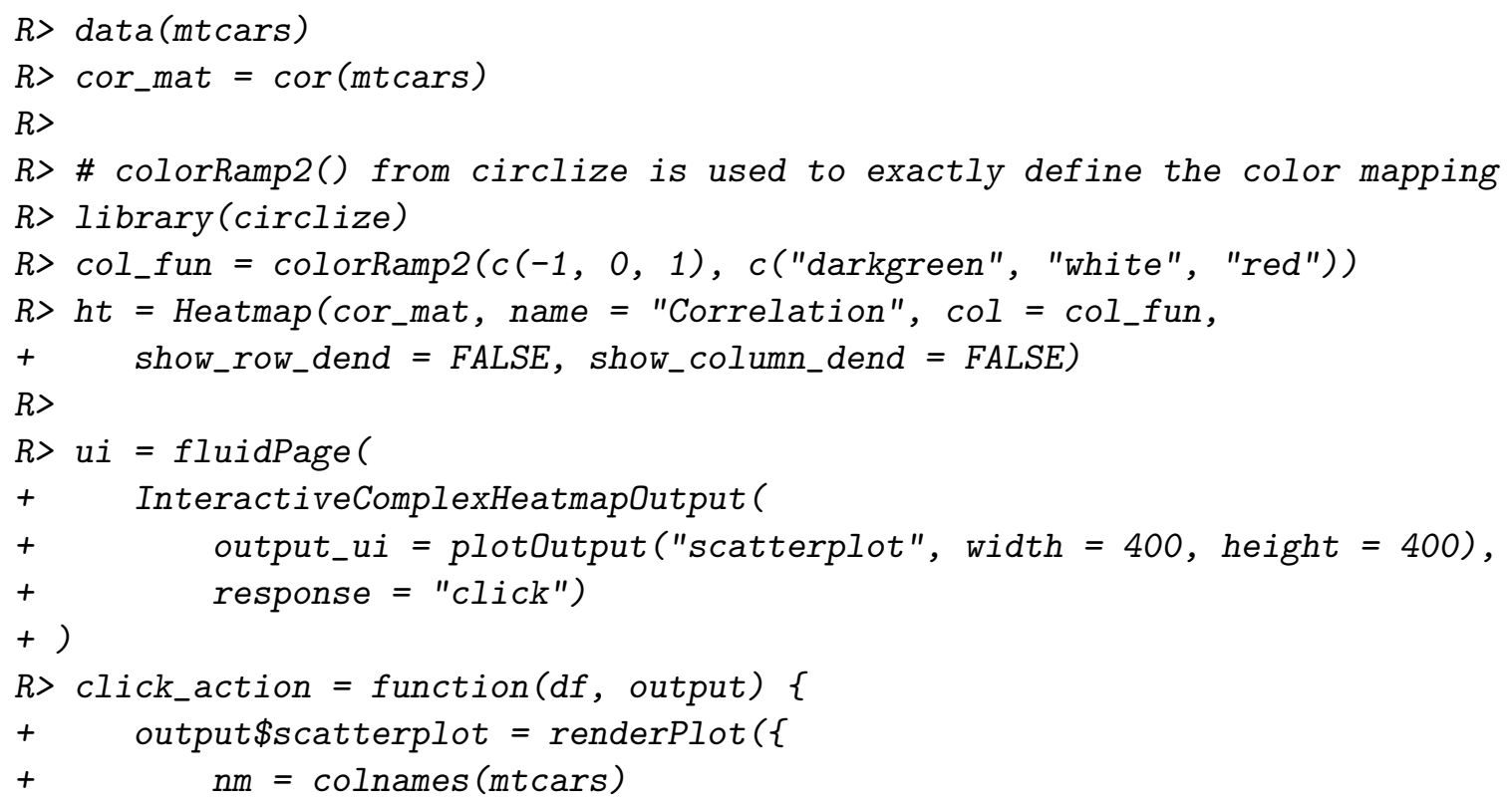




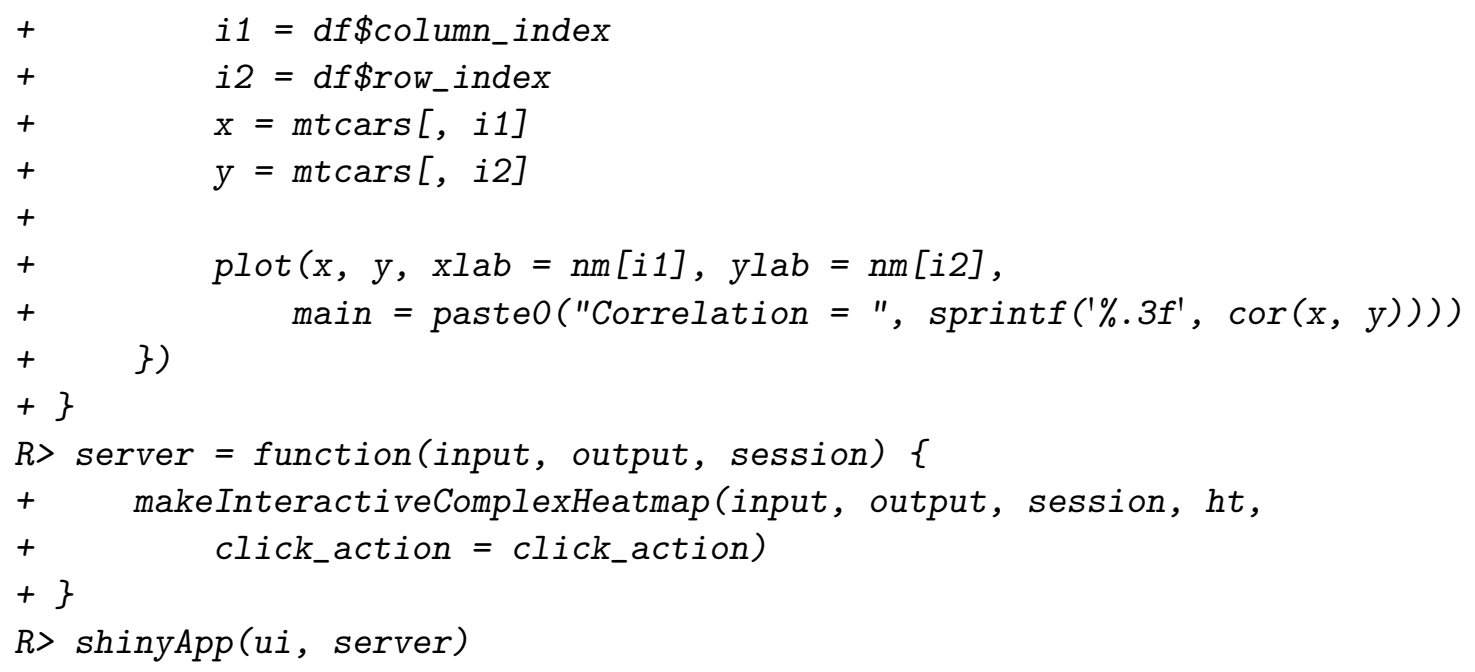

There are several other examples in InteraciveComplexHeatmap that demonstrate the use of self-defining output:

- htShinyExample(5.3): A gene expression matrix is visualized and clicking on the heatmap prints the corresponding gene ID, the link to public database and other annotations related to this gene.

- htShinyExample(5.4): The heatmap visualizes pairwise similarities of a list of Gene Ontology (GO) terms. In this example, the click and brush actions are self-defined so that the selected GO IDs as well as their detailed descriptions are printed.

- htShinyExample(5.6): The heatmap visualizes pairwise Jaccard coefficients for multiple lists of genomic regions. Clicking on the heatmap cell draws a Hilbert curve generated by the package HilbertCurve (Gu, Eils, and Schlesner 2016b) which illustrates how the two corresponding sets of genomic regions overlap.

- Section 6 demonstrates a complex example of interactively visualizing results from a differential expression analysis where the output is self-defined to link selected genes on heatmaps to a MA-plot, a volcano plot as well as a table of results only for these selected genes.

\section{Float the output}

Instead of occupying static space in the application, the output component can be floated to the mouse positions by setting argument output_ui_float = TRUE in InteractiveComplexHeatmapOutput (), then clicking, hovering or brushing on the heatmap opens a floating frame that contains the output. htShinyExample(9.1) demonstrates output floating when mouse action is set to "hover", "click" or "dblclick", respectively. If the default output is replaced by a user-defined output by assigning to argument output_ui, the self-defined output can also be floated. Figure 13 demonstrates an example where clicking on the GO similarity heatmap opens a floating frame that contains the information of the corresponding GO terms. The live version can be found in htShinyExample(9.2). 
bioRxiv preprint doi: https://doi. org/10.1101/2021.03.08 434289; this version posted May 18, 2021. The copyright holder for this preprint (which was not certified by peer review) is the author/funder, who has granted bioRxiv a license to display the preprint in perpetuity. It is made available under aCC-BY-ND 4.0 International license.

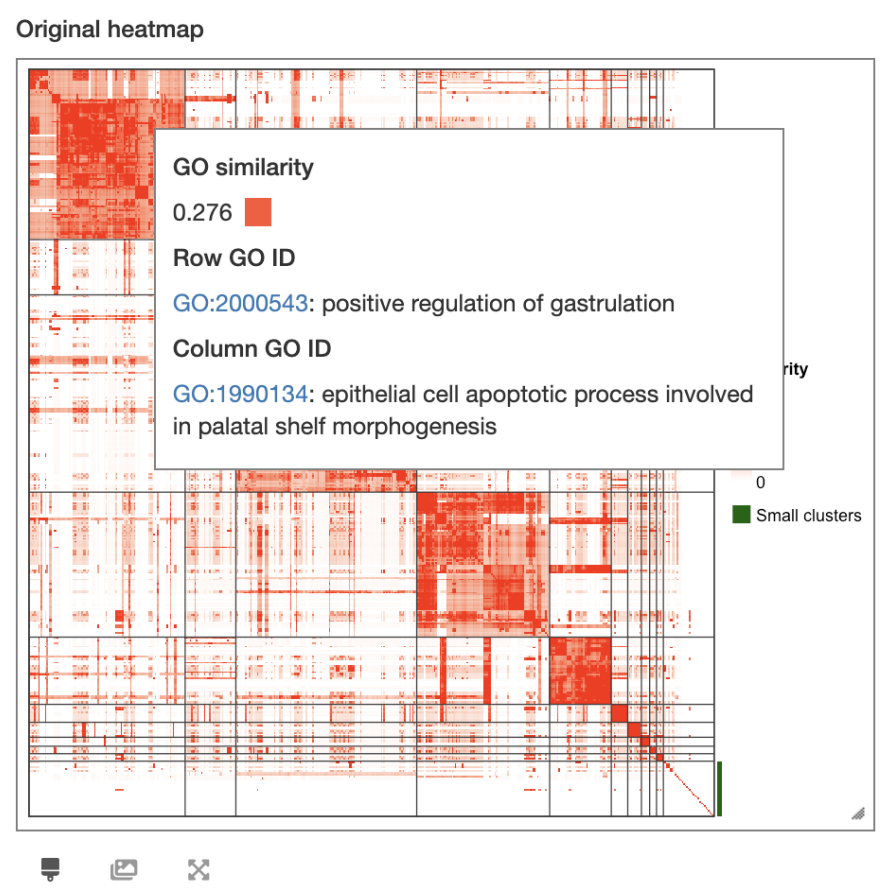

Figure 13: Floating output to mouse positions. The heatmap visualizes a GO similarity matrix from an analysis by package simplifyEnrichment. The output for clicking was self-defined to print the description of the two GO IDs and argument output_ui_float in InteractiveComplexHeatmapOutput() was set to TRUE to float the output to mouse positions. Top left of the output box corresponds to the cell that was clicked.

\section{Compact mode}

InteractiveComplexHeatmapOutput() supports a "compact mode" by setting the argument compact to TRUE, then there is only the original heatmap and the output floats at the mouse positions if hovering or clicking on heatmap. The following two lines of code are atcually identical. An example is in htShinyExample(1.11).

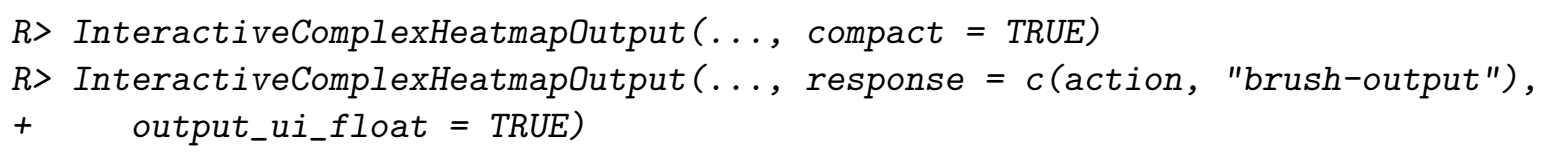

\subsection{Dynamically generate interactive heatmap widget}

In previous examples, heatmaps are already generated before generating the interactive applications. There are also scenarios where the heatmaps are generated on the fly. There might be following scenarios where heatmap is dynamically generated:

- The heatmap is based on a subset of matrix which is filtered by users, e.g., the expression matrix for differentially expressed genes filtered by different cutoffs.

- The heatmap annotations are dynamically provided by users. 
bioRxiv preprint doi: https://doi.org/10.1101/2021.03.08.434289; this version posted May 18, 2021. The copyright holder for this preprint (which was not certified by peer review) is the author/funder, who has granted bioRxiv a license to display the preprint in perpetuity. It is made available under aCC-BY-ND 4.0 International license.

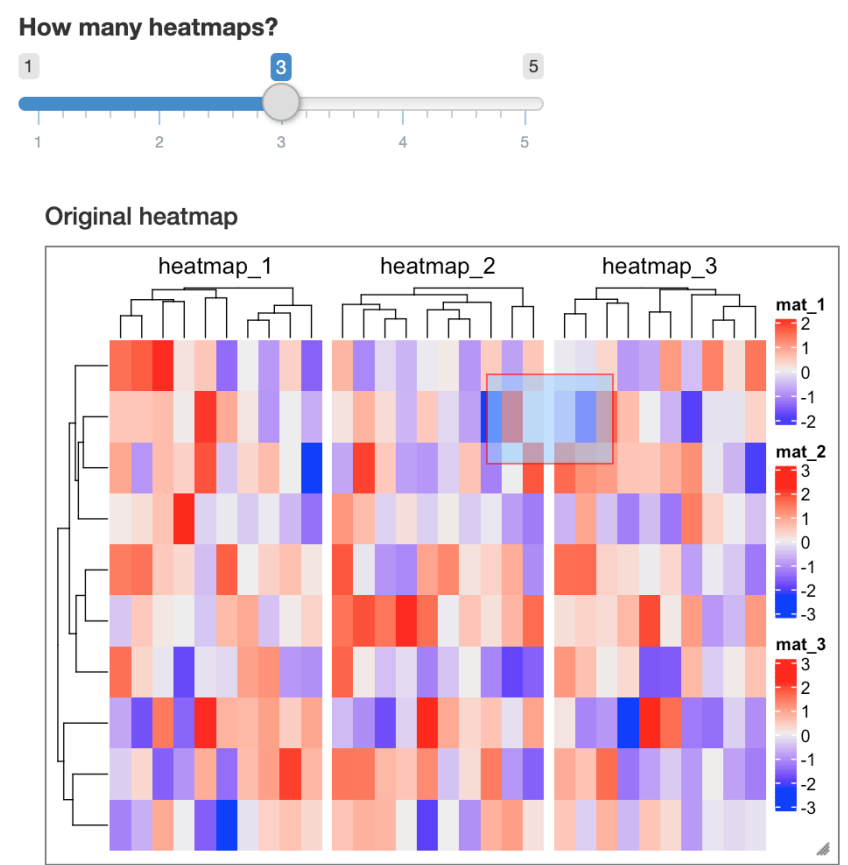

Q 둥 눙 胥
Selected sub-heatmap

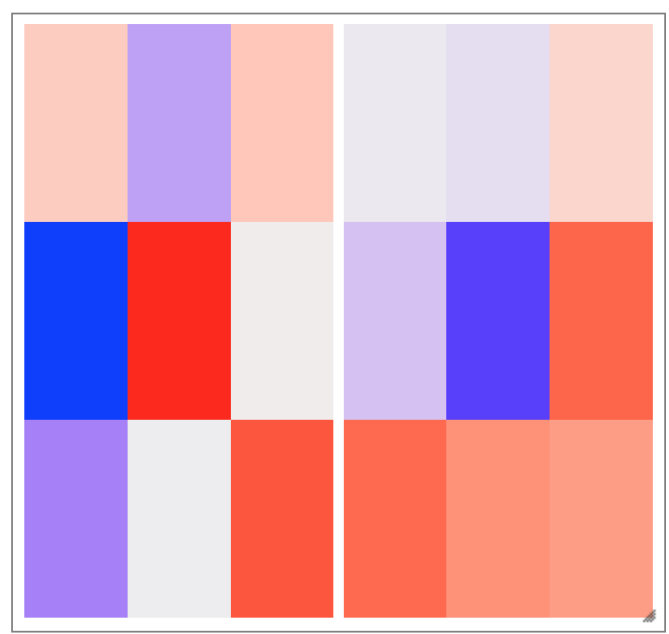

细田永

Output

Selected over 2 heatmaps with 3 rows and 6 columns. Row and column indices can be obtained by copying following code:

show/hide code

Figure 14: Dynamically generate the interactive heatmap widget. The wiget visualizes a user-specified number of heatmaps.

- The heatmap parameters are selected by users, e.g., the clustering method or the splitting variable.

- If there are multiple heatmaps, which heatmaps are going to be drawn is dynamically selected.

InteractiveComplexHeatmap supports several ways to implement dynamic interactive heatmaps.

\section{Directly use makeInteractiveComplexHeatmap()}

The use is very natural. In the next example where the interactive heatmap widget is dynamically generated according to the number of heatmaps that the user selected, makeInteractiveComplexHeatmap () is directly put inside shiny: : observeEvent () or shiny: : observe()

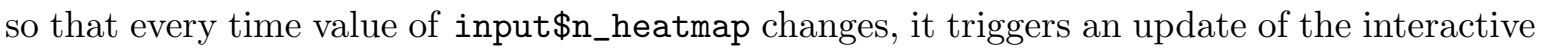
heatmap widget (Figure 14).

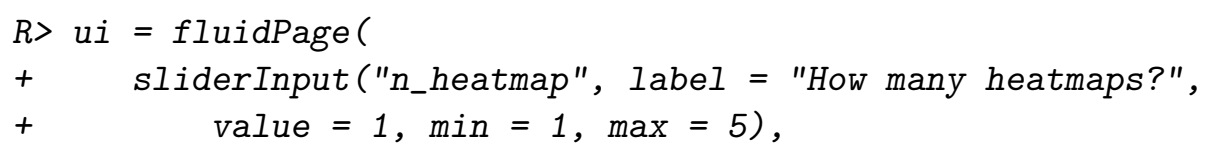




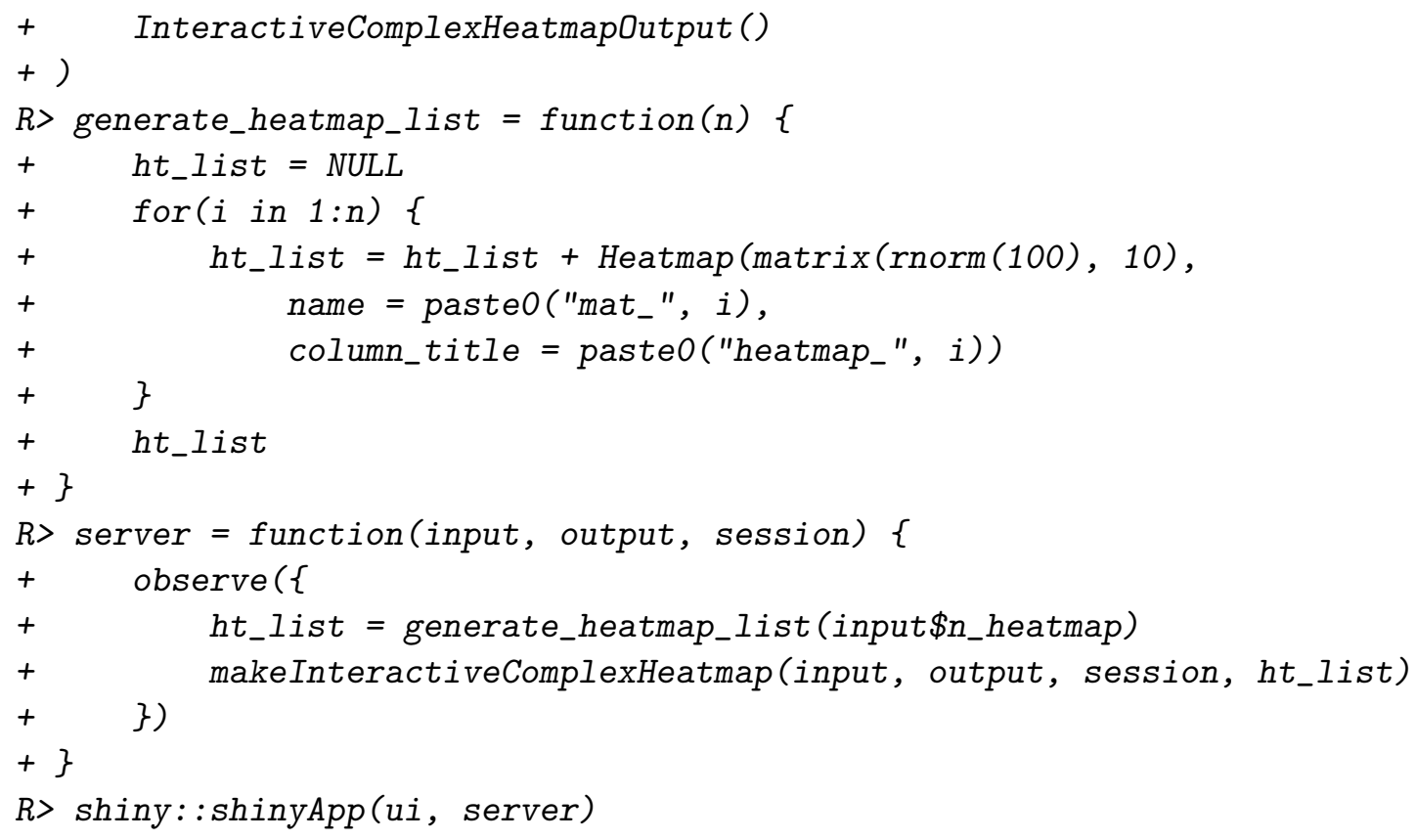

More examples can be found in htShinyExample(6.1) and htShinyExample(6.2).

\section{Use InteractiveComplexHeatmap Widget() and InteractiveComplexHeatmapModal()}

In the previous example, the UI for the interactive heatmap must be manually created and it is placed on the application permanently. The function InteractiveComplexHeatmapWidget() provides an alternative way to implement this example which dynamically creates the complete interactive heatmap widget on the fly. To use InteractiveComplexHeatmapWidget(), a placeholder by htmlOutput() should be first created on the UI (e.g., "heatmap_widget" in the following code chunk). On the server side, InteractiveComplexHeatmapWidget() dynamically creates the UI and inserts it to the place defined by htmloutput(). One advantage of using InteractiveComplexHeatmapWidget () is it supports removing the interactive heatmap widget from the application, which would be useful if the interactive widget is only for temporary use. See examples in htShinyExample (6.6) and htShinyExample(6.7).

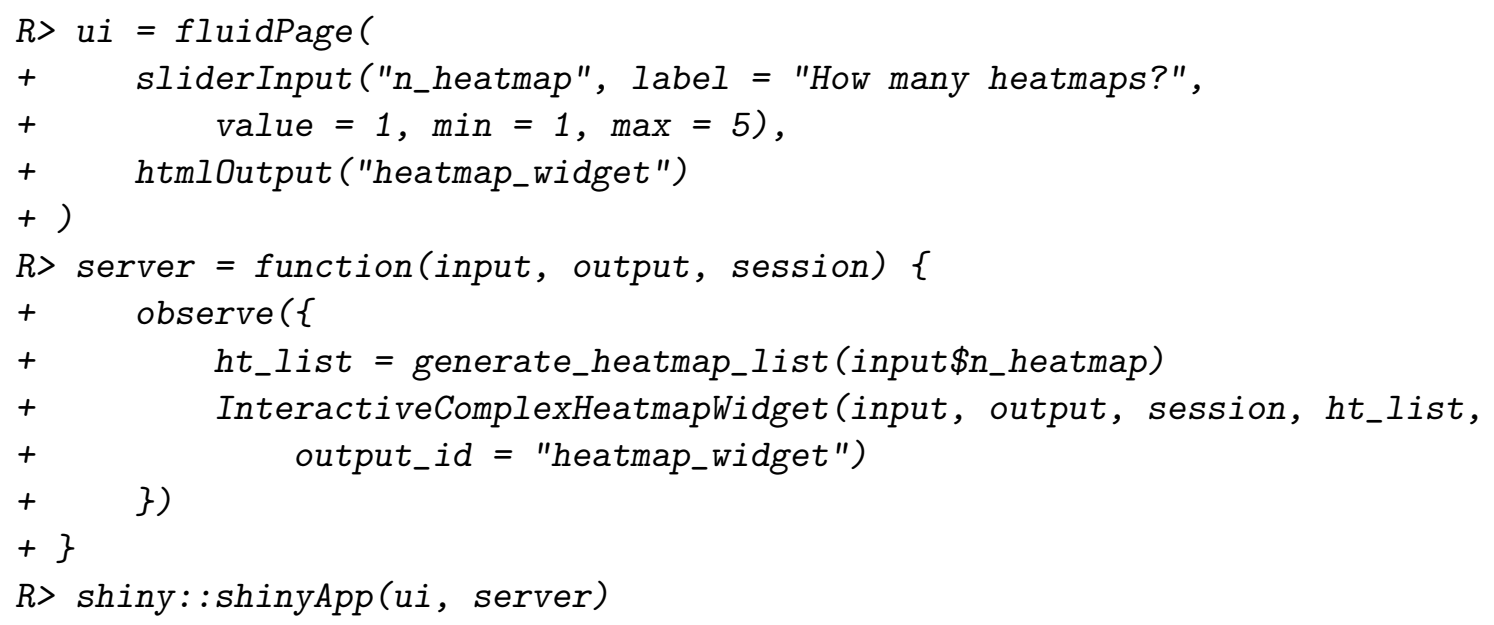


The second function InteractiveComplexHeatmapModal() is very similar as InteractiveComplexHeatmapWidget(). InteractiveComplexHeatmapModal() also dynamically generates the complete interactive heatmap widget on the fly, but it does not require to allocate a placeholder on UI, while the widget locates in a new layer above the current layer, as a so-called "modal dialog".

Following code demonstrates an example which dynamically creates an interactive widget of a numeric heatmap or a character heatmap. Live examples can be found in htShinyExample(6.3), htShinyExample(6.4) and htShinyExample(6.5). The recursive interactive heatmap introduced in Section 3.4 is also implemented with InteractiveComplexHeatmapModal ().

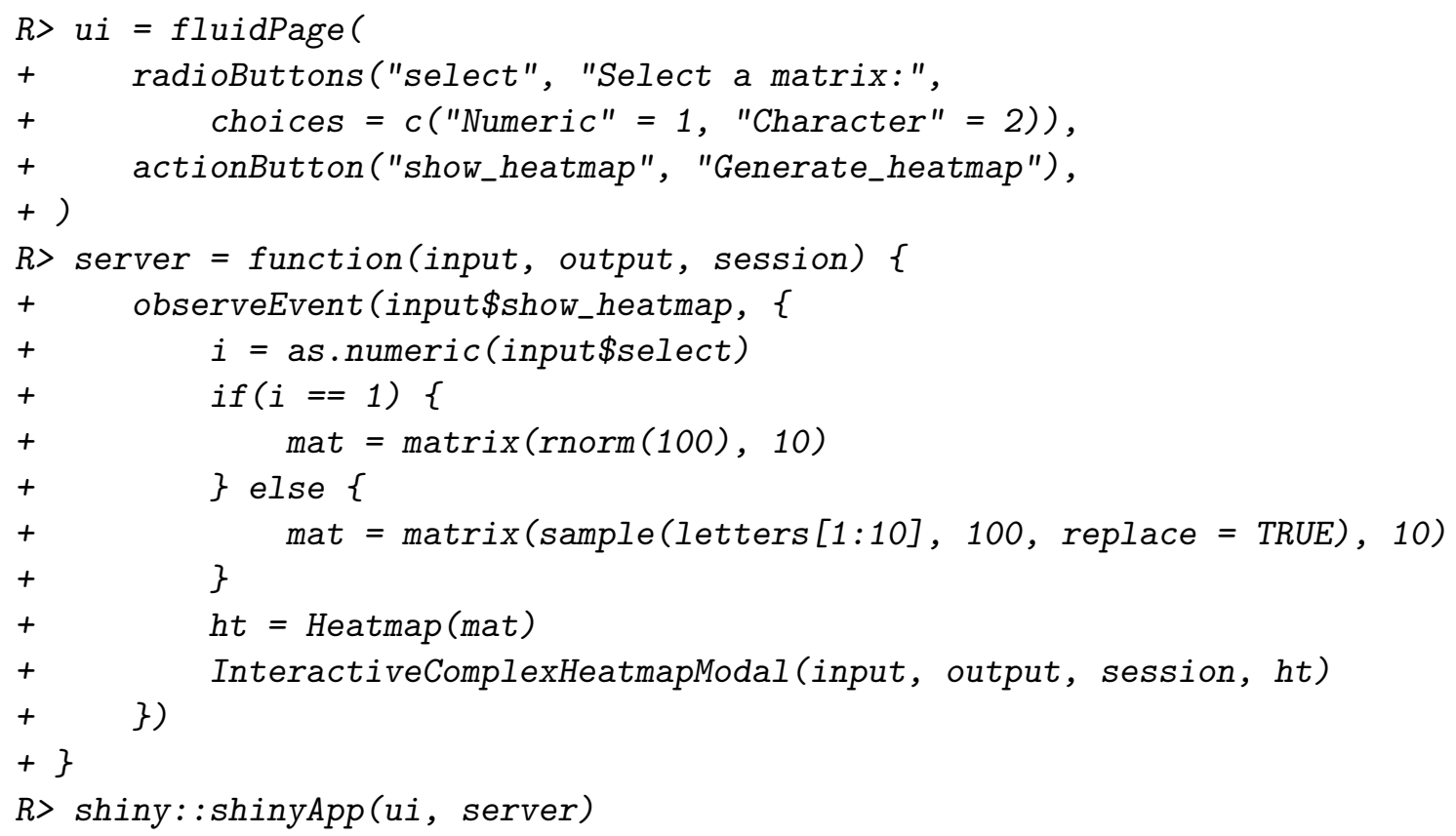

\subsection{Implement the widgets from scratch}

InteractiveComplexHeatmapOutput () and makeInteractiveComplexHeatmap () generate heatmap widgets that contain many pre-defined tools. InteractiveComplexHeatmap also provides low-level functions that directly return the information of rows and columns that were selected from heatmap so that users can define how to respond to the events on heatmap and build their own interactive heatmap widgets completely from scratch. We demonstrate the usage in the next example where ui and server are defined as follows:

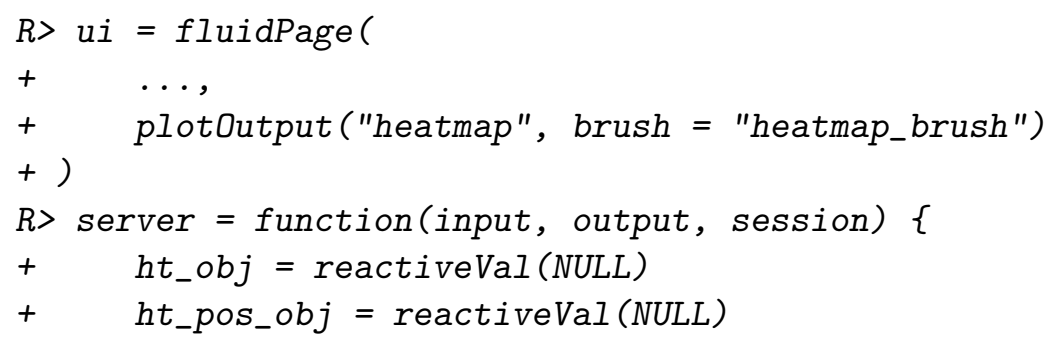


bioRxiv preprint doi: https://doi.org/10.1101/2021.03.08.434289; this version posted May 18, 2021. The copyright holder for this preprint (which was not certified by peer review) is the author/funder, who has granted bioRxiv a license to display the preprint in perpetuity. It is made available under aCC-BY-ND 4.0 International license.

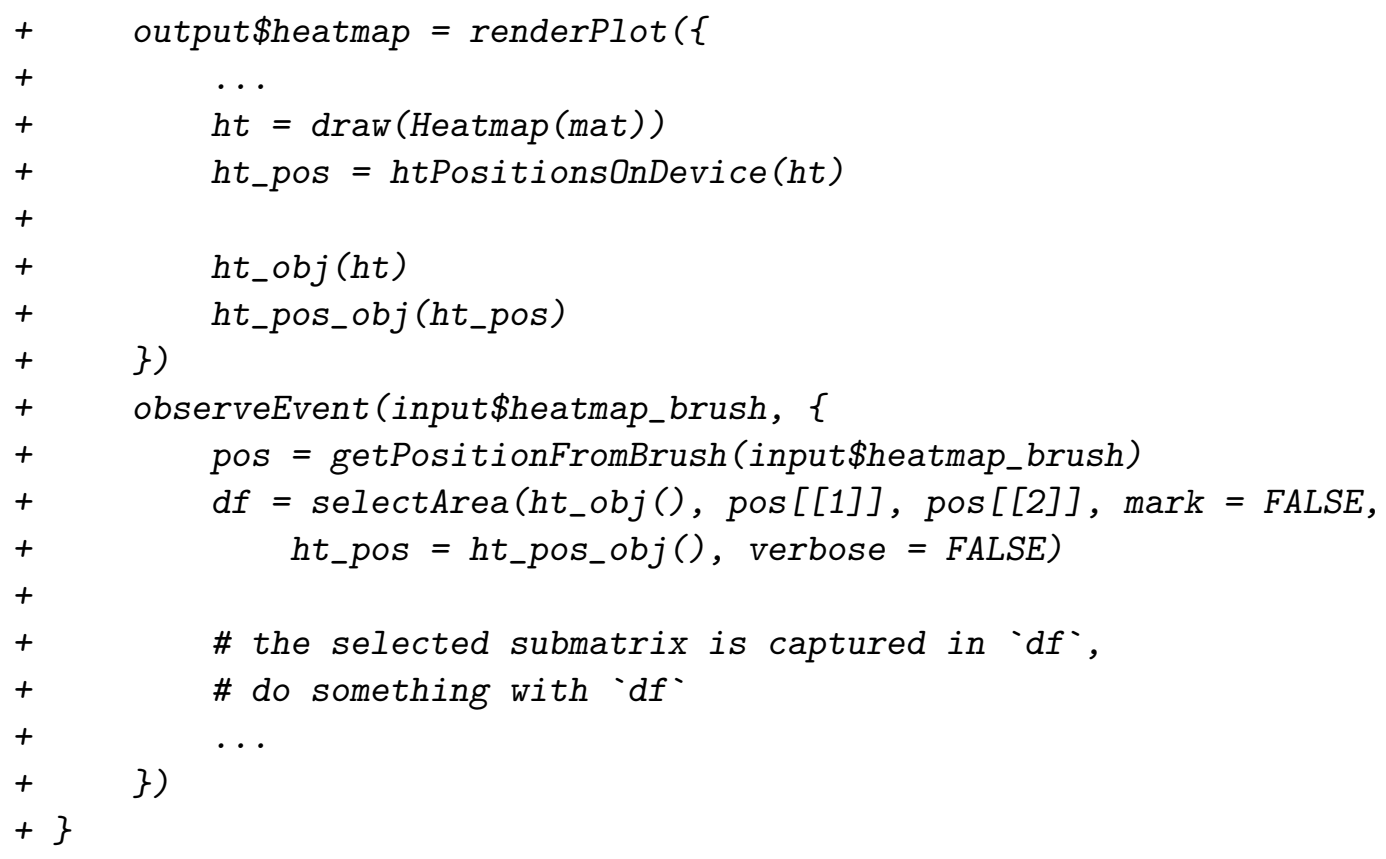

There are two points that need to be noted. 1. draw() and htPositionsOnDevice() should always be executed together and be put inside renderPlot () so that positions of all heatmap slices can be correctly calculated. 2. Use getPositionFromBrush() to retrieve the positions of the brushed area on heatmap, then the positions can be sent to selectArea() to correspond to the original matrix. Similarly, getPositionFromClick() and selectPosition() work together to retrieve row and column from the matrix that correspond to user's click on the heatmap. Runnable examples can be found in htShinyExample (5.7). This method is general that it also works for complex heatmaps, e.g., with row or column splitting, or with multiple heatmaps and annotations.

htShinyExample (5.8) demonstrates another example which visualizes a 2D density distribution. When brushing on the density heatmap, the use of makeInteractiveComplexHeatmap() which only purely visualizes a "zoomed subset" might cause a problem that is the densities in the selected sub-heatmap cannot be distinguished to background if densities are too small compared to the maximal density value from the complete dataset. To solve this problem, following the method proposed in this section, we reimplement the response of the brush event from scratch, which now triggers a new $2 \mathrm{D}$ density estimation only on the subset of the selected data. More description on this example can be found in Section 6.2.

\section{Case studies}

\subsection{Visualize differential gene expression results}

Figure 15 demonstrates an interactive heatmap application which visualizes results of differential gene expression analysis on the airway dataset performed by package DESeq2 (Love, Huber, and Anders 2014). In the application, the heatmap visualizes expression of genes that 
bioRxiv preprint doi: https://doi.org/10.1101/2021.03.08 434289; this version posted May 18, 2021. The copyright holder for this preprint (which was not certified by peer review) is the author/funder, who has granted bioRxiv a license to display the preprint in perpetuity. It is made available under aCC-BY-ND 4.0 International license.

Zuguang Gu, Daniel Hübschmann

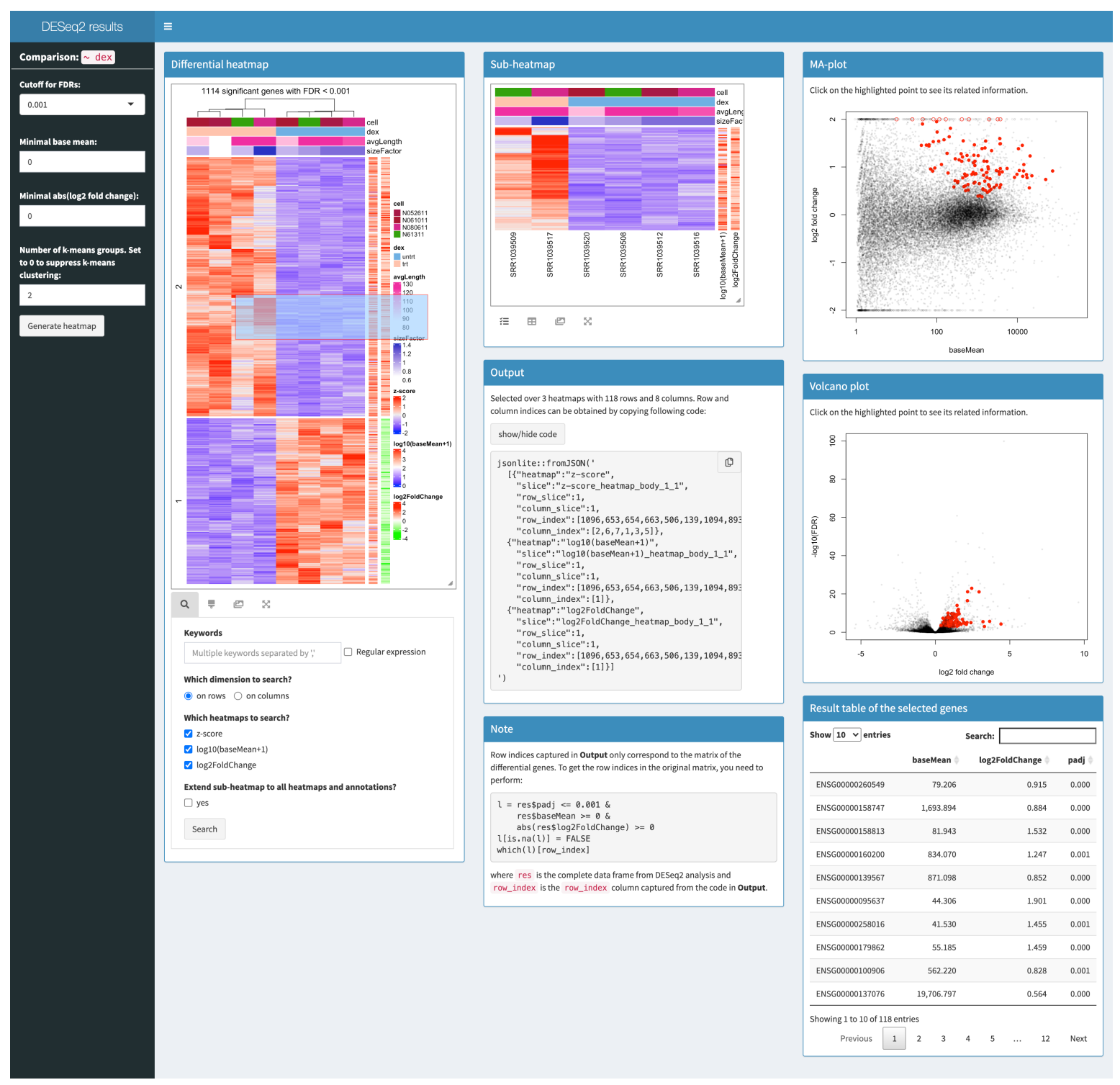

Figure 15: An interactive heatmap application for visualizing results from a differential gene expression analysis.

are significantly different from a two-condition comparison, i.e., trt vs untrt (treated vs. untreated). The application is arranged in a three-column layout where the original heatmap locates in the first column, the sub-heatmap and the default output locate in the second column, and self-defined outputs are in the third column. The heatmap components are specified separately and the layout is implemented with the shinydashboard package.

In the original heatmap list, there are two additional one-column heatmaps which visualize the absolute expression level of genes (baseMean) and the fold change between the two conditions (log2foldChange). To get more comprehensive information of how genes are differentially expressed when selecting them from heatmap, we defined extra outputs for the click and brush events on the original heatmaps. As shown in Figure 15, when a subset of genes are 

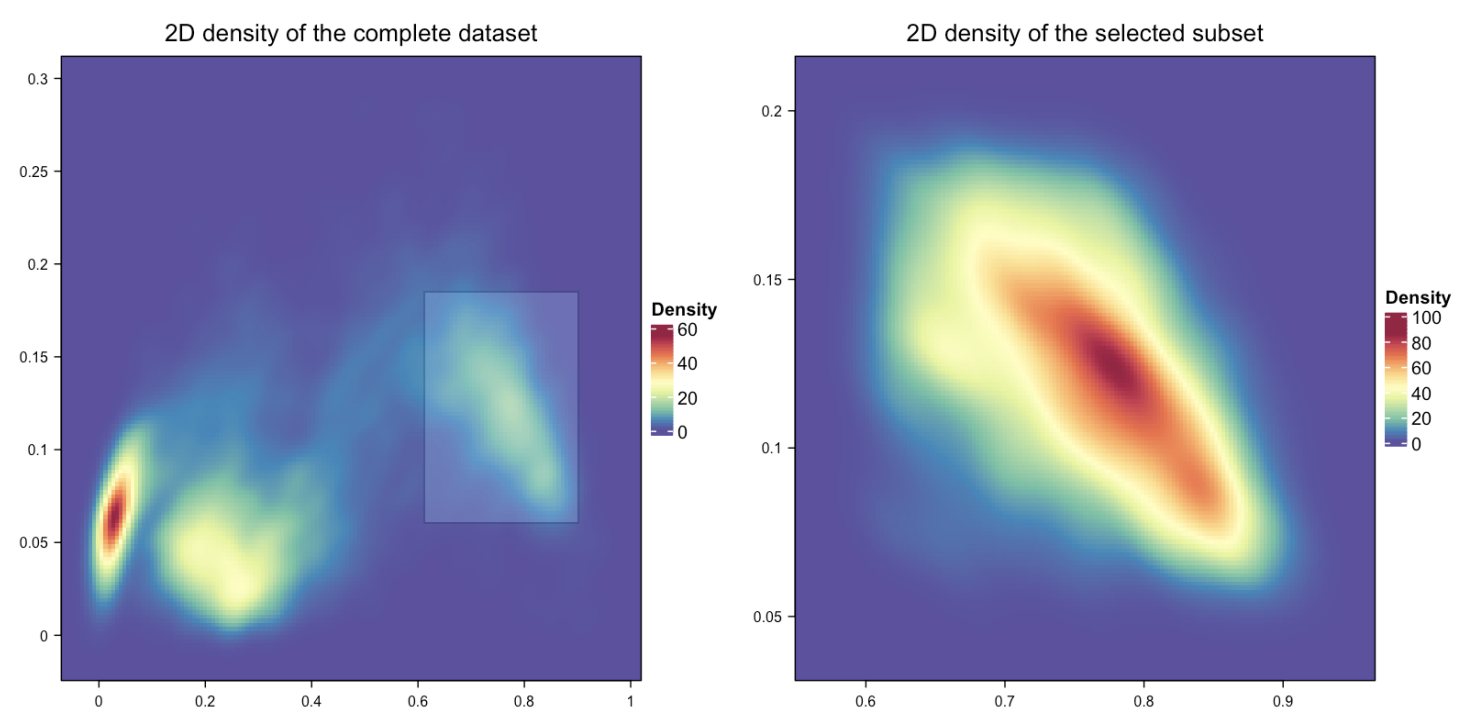

Figure 16: An interacitve visualization on a two-dimensional density distribution.

selected from the original heatmap, they are highlighted in a MA-plot which is a scatter plot of log2foldChange against baseMean where baseMean is in $\log 10$ scale and in a volcano plot which is also a scatter plot of $-\log 10$ (FDR) against log2foldchange. There is a table of statistics from DESeq2 analysis for the selected genes is printed below the two plots. Users can also search in heatmaps to obtain a subset of genes of interest to generate corresponding MA-plot, volcano plot and table.

In the left sidebar of the application, there are parameters for selecting differential genes as well as for selecting the number of groups for the $k$-means clustering applied on the expression matrix. The whole interactive widget will be updated when specific parameters are selected. The vignette "A Shiny app for visualizing DESeq2 results" in InteractiveComplexHeatmap gives a detailed explanation of implementing this Shiny application from scratch. Because differential expression analysis with DESeq2 is widely applied in transcriptomic data analysis, InteractiveComplexHeatmap implements a generic function interactivate() and a method dispatched on DESeqDataSet class which is defined by DESeq2 for storing results from differential expression analysis. Thus, interactive visualization on DESeq2 results can be simply achieved such as in the following example:

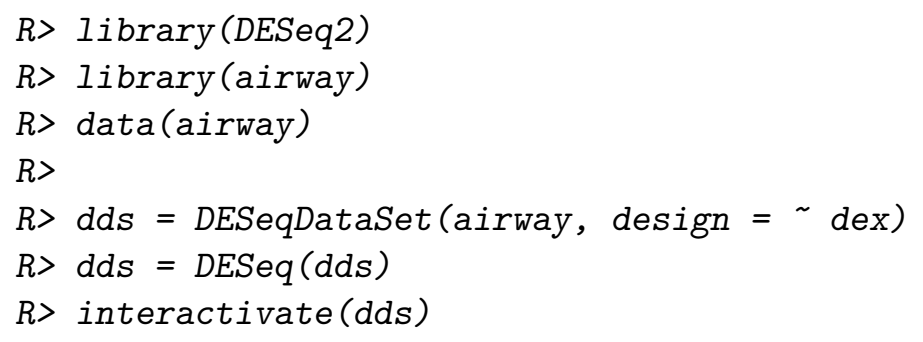

\subsection{Visualize two-dimensional density distributions}


bioRxiv preprint doi: https://doi.org/10.1101/2021.03.08.434289; this version posted May 18, 2021. The copyright holder for this preprint (which was not certified by peer review) is the author/funder, who has granted bioRxiv a license to display the preprint in perpetuity. It is made available under aCC-BY-ND 4.0 International license.

Zuguang Gu, Daniel Hübschmann

Heatmap is a popular method for visualizing two-dimensional density distributions. Nevertheless, when there are peaks that have large heights in the distribution, other peaks with smaller heights might be difficult to be distinguished from each other and even not distinguishable from background. Figure 16 implements an approach where selecting a region from the original density heatmap triggers a new two-dimensional density estimation but only on the selected subset of data. This approach helps to reveal more details in the regions which have relatively lower densities and are normally hidden in the complete dataset. To facilitate users to easier interprate two-dimensional distributions, InteractiveComplexHeatmap provides a general-purpose function interactivateDensity2D() that can be applied to two numeric vectors where the density is estimated by the package ks (Duong 2007).

$R>$ interactivateDensity2D(x,y)

If the two-dimensional density is already estimated by function ks: $: \mathrm{kde}()$, the generic function interactivate() can be directly applied.

R> fit $=k s:: k d e(\ldots)$

$R>$ interactivate( $f$ it)

\section{Conclusion}

Interactivity on heatmaps greatly facilitates users to capture features which have specific patterns on heatmaps. In this paper, we described a new R/Bioconductor package InteractiveComplexHeatmap that supports interactive visualization on complex heatmaps. It can easily export static heatmaps to interactive Shiny applications and it also provides flexible functionalities for implementing more comprehensive Shiny applications. We believe it will be a useful tool for effectively interpreting data and developmenting new tools.

\section{Acknowledgments}

This work was supported by the German Cancer Research Center-Heidelberg Center for Personalized Oncology (DKFZ-HIPO) and by the Molecular Diagnostics Program of the National Center for Tumor Diseases (NCT) Heidelberg.

\section{References}

Babicki S, Arndt D, Marcu A, Liang Y, Grant JR, Maciejewski A, Wishart DS (2016). "Heatmapper: web-enabled heat mapping for all." Nucleic Acids Research, 44(W1), W147W153. doi:10.1093/nar/gkw419.

Barter RL, Yu B (2018). "Superheat: An R Package for Creating Beautiful and Extendable Heatmaps for Visualizing Complex Data." Journal of Computational and Graphical Statistics, 27(4), 910-922. doi:10.1080/10618600.2018.1473780. 
bioRxiv preprint doi: https://doi org/10.1101/2021.03.08.434289; this version posted May 18, 2021. The copyright holder for this preprint (which was not certified by peer review) is the author/funder, who has granted bioRxiv a license to display the preprint in perpetuity. It is made available under aCC-BY-ND 4.0 International license.

Chang W, Borges Ribeiro B (2018). shinydashboard: Create Dashboards with 'Shiny'. R package version 0.7.1, URL https://CRAN.R-project.org/package=shinydashboard.

Chang W, Cheng J, Allaire J, Sievert C, Schloerke B, Xie Y, Allen J, McPherson J, Dipert A, Borges B (2021). shiny: Web Application Framework for R. R package version 1.6.0, URL https://CRAN.R-project.org/package=shiny.

Cheng J, Galili T (2018). d3heatmap: Interactive Heat Maps Using 'htmlwidgets' and 'D3.js'. R package version 0.6.1.2, URL https://CRAN.R-project.org/package=d3heatmap.

Conway JR, Lex A, Gehlenborg N (2017). "UpSetR: an R package for the visualization of intersecting sets and their properties." Bioinformatics, 33(18), 2938-2940. doi:10.1093/ bioinformatics/btx364.

Duong T (2007). "ks: Kernel Density Estimation and Kernel Discriminant Analysis for Multivariate Data in R." Journal of Statistical Software, Articles, 21(7), 1-16. ISSN 15487660. doi:10.18637/jss.v021.i07. URL https://www.jstatsoft.org/v021/i07.

Eisen MB, Spellman PT, Brown PO, Botstein D (1998). "Cluster analysis and display of genome-wide expression patterns." Proceedings of the National Academy of Sciences, 95(25), $14863-14868$.

Fernandez NF, Gundersen GW, Rahman A, Grimes ML, Rikova K, Hornbeck P, Ma'ayan A (2017). "Clustergrammer, a web-based heatmap visualization and analysis tool for highdimensional biological data." Scientific Data, 4(1), 170151. ISSN 2052-4463. doi:10.1038/ sdata.2017.151.

Galili T, O'Callaghan A, Sidi J, Sievert C (2017). "heatmaply: an R package for creating interactive cluster heatmaps for online publishing." Bioinformatics, 34(9), 1600-1602. doi: 10.1093/bioinformatics/btx657.

Gao J, Aksoy BA, Dogrusoz U, Dresdner G, Gross B, Sumer SO, Sun Y, Jacobsen A, Sinha R, Larsson E, Cerami E, Sander C, Schultz N (2013). "Integrative Analysis of Complex Cancer Genomics and Clinical Profiles Using the cBioPortal." Science Signaling, 6(269), pl1-pl1. doi:10.1126/scisignal.2004088.

Gaujoux R, Seoighe C (2010). "A flexible R package for nonnegative matrix factorization." BMC Bioinformatics, 11(1), 367. doi:10.1186/1471-2105-11-367.

Gu Z (2020). Rasterization in ComplexHeatmap. URL https://jokergoo.github.io/2020/ 06/30/rasterization-in-complexheatmap/.

Gu Z (2021). ComplexHeatmap Complete Reference. URL https://jokergoo.github.io/ ComplexHeatmap-reference/book/.

Gu Z, Eils R, Schlesner M (2016a). "Complex heatmaps reveal patterns and correlations in multidimensional genomic data." Bioinformatics, 32(18), 2847-2849. doi:10.1093/ bioinformatics/btw313.

Gu Z, Eils R, Schlesner M (2016b). "HilbertCurve: an R/Bioconductor package for highresolution visualization of genomic data." Bioinformatics, 32(15), 2372-2374. doi:10. 1093/bioinformatics/btw161. 
bioRxiv preprint doi: https://doi org/10.1101/2021.03.08.434289; this version posted May 18,2021 . The copyright holder for this preprint (which was not certified by peer review) is the author/funder, who has granted bioRxiv a license to display the preprint in perpetuity. It is made available under aCC-BY-ND 4.0 International license.

Zuguang Gu, Daniel Hübschmann

Gu Z, Eils R, Schlesner M, Ishaque N (2018). "EnrichedHeatmap: an R/Bioconductor package for comprehensive visualization of genomic signal associations." BMC Genomics, 19(1), 234. doi:10.1186/s12864-018-4625-x.

Gu Z, Hübschmann D (2021). "simplifyEnrichment: an R/Bioconductor package for Clustering and Visualizing Functional Enrichment Results." bioRxiv. doi:10.1101/2020.10.27. 312116.

Gu Z, Schlesner M, Hübschmann D (2020). "cola: an R/Bioconductor package for consensus partitioning through a general framework." Nucleic Acids Research, 49(3), e15-e15. doi: 10.1093/nar/gkaa1146.

Hahsler M, Hornik K, Buchta C (2008). "Getting Things in Order: An Introduction to the R Package seriation." Journal of Statistical Software, Articles, 25(3), 1-34. doi: 10.18637/jss.v025.i03.

Kiselev VY, Kirschner K, Schaub MT, Andrews T, Yiu A, Chandra T, Natarajan KN, Reik W, Barahona M, Green AR, Hemberg M (2017). "SC3: consensus clustering of single-cell RNA-seq data." Nature Methods, 14(5), 483-486. doi:10.1038/nmeth.4236.

Kolde R (2019). pheatmap: Pretty Heatmaps. R package version 1.0.12, URL https://CRAN. $\mathrm{R}$-project.org/package=pheatmap.

Love MI, Huber W, Anders S (2014). "Moderated estimation of fold change and dispersion for RNA-seq data with DESeq2." Genome Biology, 15(12), 550. doi:10.1186/ s13059-014-0550-8.

Morpheus (2021). Versatile matrix visualization and analysis software. URL https: //software.broadinstitute.org/morpheus.

Pagès H, Lawrence M, Aboyoun P (2020). S4Vectors: Foundation of vector-like and list-like containers in Bioconductor. R package version 0.26.1.

R Core Team (2020). R: A Language and Environment for Statistical Computing. R Foundation for Statistical Computing, Vienna, Austria. URL https://www.R-project.org/.

Ryan M, Stucky M, Wakefield C, Melott J, Akbani R, Weinstein J, Broom B (2019). "Interactive Clustered Heat Map Builder: An easy web-based tool for creating sophisticated clustered heat maps." F1000Research, 8(1750). doi:10.12688/f1000research.20590.1.

Schep AN, Kummerfeld SK (2017). "iheatmapr: Interactive complex heatmaps in R." Journal of Open Source Software, 2(16), 359. doi:10.21105/joss.00359.

Warnes GR, Bolker B, Bonebakker L, Gentleman R, Huber W, Liaw A, Lumley T, Maechler M, Magnusson A, Moeller S, Schwartz M, Venables B (2020). gplots: Various R Programming Tools for Plotting Data. R package version 3.1.0, URL https://CRAN.R-project. org/package $=$ gplots.

Wilke CO (2020). gridtext: Improved Text Rendering Support for 'Grid' Graphics. R package version 0.1.1, URL https://CRAN.R-project.org/package=gridtext. 
Wilkinson L, Friendly M (2009). "The History of the Cluster Heat Map." The American Statistician, 63(2), 179-184. doi:10.1198/tas.2009.0033.

Zhao S, Yin L, Guo Y, Sheng Q, Shyr Y (2021). heatmap3: An Improved Heatmap Package. R package version 1.1.9, URL https://CRAN.R-project.org/package=heatmap3.

\author{
Affiliation: \\ Zuguang Gu \\ German Cancer Research Center \\ Computational Oncology, Molecular Diagnostics Program, National Center for Tumor Dis- \\ eases $(\mathrm{NCT})$ \\ and \\ DKFZ-HIPO (Heidelberg Center for Personalized Oncology) \\ Im Neuenheimer Feld 280 \\ 69120 Heidelberg, Germany \\ E-mail: z.gu@dkfz-heidelberg.de \\ URL: http://jokergoo.github.io \\ Daniel Hübschmann \\ German Cancer Research Center \\ Computational Oncology, Molecular Diagnostics Program, National Center for Tumor Dis- \\ eases $(\mathrm{NCT})$ \\ and \\ Heidelberg Institute of Stem cell Technology and Experimental Medicine (HI-STEM) \\ and \\ German Cancer Consortium (DKTK) \\ Im Neuenheimer Feld 280 \\ 69120 Heidelberg, Germany \\ and \\ Department of Pediatric Immunology, Hematology and Oncology, University Hospital Heidel- \\ berg \\ 69120 Heidelberg, Germany \\ E-mail: d.huebschmann@dkfz-heidelberg.de
}

